\title{
Biocompetent nanorobots can transfer chemical energy into mechanical work even in ionic environments
}

\begin{abstract}
Since enzyme-powered micro/nanomotors have demonstrated excellent biocompatibility and bioavailability, as well as the ability to transform chemical energy into mechanical work even in ionic settings, they have sparked considerable interest in the field of hybrid micro/nanomotors. They have enabled active targeting, cargo loading and release, and biosensing, all of which are made feasible by the complex design and functionalization of their devices. Comprehensive understanding of structural complementary domains and synergistic interactions will provide unique characteristics for the bottom-up self-assembling of numerous enzymes in the development of bioinspired biomolecular machines in future concepts, such as the development of bioinspired biomolecular machines. The movement of enzyme-powered nanorobots will be controlled by a concentration gradient in a certain direction. If they are used in medical applications, they will move in reaction to a chemical gradient generated at the site of tissue damage or inflammation. CAT-powered poly (lactic-co-glycolic acid) micromotors that move in response to a hydrogen peroxide concentration gradient for the delivery of a periodontal disease treatment were recently published in a study by Wilson and colleagues. 64 In an in vitro model of inflammatory periodontitis, a phorbol ester activated macrophage cell was employed to investigate the chemotactic self-propulsion of micromotors.
\end{abstract}




\section{Introduction}

Molecular motor proteins (kinesins, dyneins, and myosins) are natural surface walkers in animal cells that use the energy from ATP hydrolysis to travel along polarized cytoskeletal filaments. 1 Scientists have used enzyme biomolecules as catalytic engines to power variably shaped artificial micro-/nanomotors as a result of these motor proteins. 2 Enzymes are proteins that have the catalytic capacity to rapidly transform substrates into products in biological systems. Because of the heat produced during substrate turnover, enzyme macromolecules have increased diffusion at the molecular level. 3 Molecule motility is thought to be caused by local and global heat effects, phoresis, and changes in enzyme conformations, and the processes outside the catalysis-motion connection are currently being debated. 5 Furthermore, when utilizing a microfluidic device with a substrate gradient, enzymatic macromolecules conduct chemotaxis because their increased diffusion occurs in a concentration-dependent manner, and the same behavior has been seen for enzyme-powered micromotors. 7

Enzyme engines have several advantages over conventional engines, including improved performance for hydrogen peroxide decomposition, motion rate sensing of environmental pollutants, and, most importantly, force generation via local chemical conversion of glucose or urea naturally occurring in biofluids. The glucose content in blood serum is typically around $5.5 \mathrm{~mm} 10$, while the concentration in saliva is believed to be in the micromolar range (8-210 m11). About 15 years ago, artificial enzyme-powered motors using glucose as the main fuel were created. Carbon-based structures with an immobilized pair of redox enzymes pushed by the impetus of a hydrated proton stream and oxygen bubble generation were created by Mano and Heller12, and Ferringa et al.13, respectively. Urea can be found in human saliva $(3-10 \mathrm{~mm})$, human blood $(2.6-6.5 \mathrm{~mm}$ ), and human urine (up to a 50-fold greater content with significant variation). 15 Nanomotors that use urea as a fuel have been demonstrated to transport doxorubicin to cancer cells more efficiently than a passive delivery system and to be $\mathrm{pH}$-responsive after being modified with DNA nanoswitches. 17

This is a review of existing enzyme-powered micro/nanomotors as well as recent proof-of-concept applications in this paper. The tubular, spherical, and Janus-like micro/nanomotors, as well as those based on supramolecular stomatocytes and metal-organic frameworks, are grouped together (MOFs).

\section{Micro/Nanomotor Propulsion Using Two Enzymes}

Despite the large number of enzymes accessible, only a handful are used as catalytic engines to power micro/nanomotors. They are primarily redox enzymes, and they have attracted a lot of scientific attention as electrocatalytic components of biosensors, bioreactors, and biofuel cells. $22 \mathrm{Micro} /$ nanomotors powered by catalytic hydrogen peroxide reactions have received a lot of attention in the field of micro/nanotechnology. 
The disproportionation of $\mathrm{H} 2 \mathrm{O} 2$ to water and oxygen occurs in the CAT reaction. With the capacity to breakdown more than one million $\mathrm{H} 2 \mathrm{O} 2$ molecules per molecule of enzyme, CAT is recognized to have one of the highest enzymatic turnover rates. If $\mathrm{H} 2 \mathrm{O} 2$ is accessible as a unique substrate in a neutral solution, HRP catalyzes the H2O2-mediated oxidation of different substrates and produces oxygen in a CAT-like manner. 24

GOx produces $\mathrm{H} 2 \mathrm{O} 2$ by oxidizing gluconic acid with glucose. Because of the toxicity of $\mathrm{H} 2 \mathrm{O} 2$ and the low activity of GOx, the GOx-CAT pair was utilized to power micro/nanomotors via cascade catalytic processes that started with glucose and ended with oxygen bubbles and water.

A soluble enzyme directly in solution can catalyze the catalytic synthesis of the necessary fuel in situ, in addition to a multienzyme ensemble coupled to micro/nanomotors. This was accomplished by using XOD to catalyze the oxidation of hypoxantine to produce radical anions (2 $\mathrm{O} 2)$, as well as $\mathrm{H} 2 \mathrm{O} 2.25$

Hydrolytic enzymes, which have been extensively investigated as biochemical catalysts in medicine, biosensing, and bioremediation26, were used to power micro-/nanomotors in order to address the issue of both biocompatible fuel and its turnover products. UR (urea amidohydrolase) is one of them, and it is involved in chemotactic motility. The hydrolysis of urea to generate ammonia and carbamate, which are then decomposed into ammonia and carbon dioxide, is catalyzed by this nickel-dependent metalloenzyme. 27 At $10 \mathrm{~mm}$ urea, immobilization of a multilayered assembly of bionylated UR on the micromotors resulted in increased propulsion at speeds of up to $(21.50 .8) \mathrm{m} \mathrm{s} 1.28$ The fact that ammonia generated during urea conversion has a cytotoxic impact at concentrations higher than $50 \mathrm{~mm}$ (total conversion of $25 \mathrm{~mm}$ urea) is a significant limitation of UR-powered micromotors. 16

Lipases, as a triglyceride hydrolysis catalyst, can serve a dual purpose in micromotors: 1) they can create thrust by degrading triglyceride-based fuel, and 2) they can decrease high plasma triglyceride levels. 29 Lipases should be chosen for their substrate specificity and positioning preference for building highly effective micro-/nanomotors. One potential drawback of using trypsin as a catalytic component of micro-/nanomotors 30 is its limited selectivity for extracellular proteins, which limits the number of peptide substrates accessible.

\section{Micro/Nanomotors Powered by Enzymes}

To prevent zero net forces, active self-propulsion of enzyme-powered micro-/nanomotors requires an asymmetric distribution of enzyme biomolecules, 31 while an appropriate quantity of immobilized enzyme on the micro-/nanomotors is required to counteract intrinsic Brownian motion. Furthermore, it has been demonstrated that when the same micromotor is fitted with a different enzyme, the resultant motion dynamics are determined by the reaction kinetics of the reaction on a specific substrate.32 Micro/nanomotors powered by enzymes with a high turnover rate exhibit rapid active motion in theory, demonstrating the link between intrinsic enzyme characteristics and propulsion performance. 33 
The majority of enzyme-powered micro/nanomotors are spherical capsules, spheres, or tubular shapes. Brownian motion begins to have a major influence on the resultant motion dynamics when the size of artificial motors is reduced to submicron dimensions. As a result of the rapid rotating diffusion, the directionality of motion is lost, and the nanomotors exhibit increased diffusion. 34 The propulsion of enzyme-powered micro-/nanomachines is usually explained by two distinct processes, according to the scientists. 35 First, relatively big particles and tubular geometries frequently use bubble-induced propulsion. It is based on the formation of gas bubbles at the solid-liquid interface, generally with traces of a surfactant present. The motion of micromotors is aided by the emission of gas bubbles, which produce a bubble recoil push. The second process is self-phoresis, in which enzymatic activity generates local concentration 36 or electrical potential gradients over the surface of a motor with submicron or nanoscale dimensions.

Furthermore, several enzymes have been found to engage in chemotaxis, or directed movement toward areas with high substrate concentrations. 38 Thermodynamically advantageous binding interactions between the active site of an enzyme biomolecule and a substrate are thought to be the source of enzyme chemotaxis. Expulsion of water molecules from enzyme water pockets, in combination with binding and catalysis, causes conformational changes in biomolecules, which result in hydrodynamic fluxes, and therefore enzyme-linked particle propulsion. $38 \mathrm{~b}, 39,5$

Sen et al. recently hypothesized that for liposomes coated with enzymes in microfluidic channels, there was an interaction between enzyme-catalysis-induced positive chemotaxis and solute-phospholipid-based negative chemotaxis. 40 In a gradient using $\mathrm{H} 2 \mathrm{O} 2$ as a substrate, CAT-coated liposomes showed positive chemotaxis. In the presence of an ATP gradient, ATPase-linked liposomes displayed positive chemotaxis, but in the presence of the reaction products adenosine diphosphate (ADP) and phosphate ion (Pi), they showed negative chemotaxis. Negative chemotaxis of UR-coated liposomes away from the substrate was observed. Surprisingly, the opposite behavior has been described in the literature for a single UR enzyme, with both chemotaxis toward urea 6 and antichemotaxis away from urea. 5 Golestanian et al. sought to explain experimental findings on UR, claiming that enzyme chemotaxis was governed by a struggle between diffusiophoresis and binding-induced changes in the enzyme's diffusion coefficient. 41 Above a threshold substrate concentration, phosis takes precedence, whereas binding-induced changes in diffusion take precedence at lower concentrations.

\section{Tubular Micro/Nanomotors Powered by Enzymes}

Bubble-driven tubular micromotors collect energy from chemical processes taking place inside a tube or cone's interior cavity. $42 \mathrm{Schmidt}$ s team formed Ti/Au micromotors $(2 \mathrm{~m}$ long) with CAT immobilized on the inner $\mathrm{Au}$ layer in 2010. At 1.5 percent $\mathrm{H} 2 \mathrm{O} 2$ concentration, these microjets had a velocity of $226 \mathrm{~m} \mathrm{~s} 1.8$ 
Wang et al. formed bilayer PEDOT/Au-CAT microtubes $(8-20 \mathrm{~m}$ long) using a similar design, which significantly reduced their motion velocity in the presence of enzyme inhibitors like $\mathrm{Hg}$, pesticides, and herbicides, 9, as well as the sarin simulant diethyl chlorophosphate (DCP) in the surrounding environment. 20

Polymer-based tubular micromotors with a thermoresponsive gelatin layer holding $\mathrm{Au}$ nanoparticles (NPs), CAT, and doxorubicin were produced to generate a stimulus response for cargo release. At 0.5 percent $\mathrm{H} 2 \mathrm{O} 2$ in cell culture conditions and at physiological temperature, the micromotors ( $20 \mathrm{~m}$ long) were bubble driven at a velocity of $4 \mathrm{~m} \mathrm{~s} 1$. The gel-sol phase transition of the gelatin hydrogel caused by the photothermal action of Au NPs under near-infrared (NIR) irradiation triggered doxorubicin release. 19

Wang's group developed fully biological millimeter-sized motors with built-in CAT and peroxidase activities. Motors made from sections of the radish Raphanus sativus push themselves into a solution of $\mathrm{H} 2 \mathrm{O} 2$ (5\%) while simultaneously decomposing different phenolic compounds as pollutants. 18

Tubular-silica-based nanomotors (200 nm diameter) with UR immobilized within were constructed and demonstrated longitudinal self-propulsion in the presence of urea $(100 \mathrm{~mm})$ in addition to CAT/H2O2-based bubble-propelled systems. 21 These nanotube-based motors are said to provide directed motion by using a self-generated concentration gradient. Nanotubes containing UR immobilized on their outer surface were unable to overcome Brownian motion, confirming this hypothesis.

\section{Spherical and Janus-like Micro/Nanomotors Powered by Enzymes}

\subsection{Systems based on mesoporous silicon}

Mesoporous materials have garnered a lot of interest in the creation of spherical structures for a variety of applications, ranging from nanocatalysis to energy storage and conversion to healthcare. 43 Spherical mesoporous-silica-based particles, for example, have a limited size distribution, an ordered porous structure, a high pore volume and surface area, and, most importantly, an adjustable surface chemistry that allows biomolecules to be immobilized. 44 The CAT enzyme and single-stranded DNA were chemically conjugated and fixed on opposing sides of mesoporous silica particles (MSPs). The resultant micromotors (500-700 $\mathrm{nm}$ ) were demonstrated to sense and collect compatible oligonucleotide fragments in biological samples that are self-propelled by bubble thrust at $2.5 \mathrm{wt} \mathrm{H} 2 \mathrm{O} 2.45$ The existence of holes or cavities on the surface of spherical micromotors is ideal for the nucleation of gas molecules. Sanchez and Ma created a Janus mesoporous silica cluster (JMSC) composed of nanoparticles with chemically attached CAT, based on the assumption of a rough surface. 46 Magnetically guided micromotors were produced by half-coating the JMSC with nickel and achieving thrust from oxygen bubbles at $1.5 \mathrm{wt} \mathrm{H} 2 \mathrm{O} 2$. 
Chemical conjugation of CAT, UR, and an ensemble of CAT/GOx or CAT/UR/GOx resulted in a number of MSP-based micro- and nanomotors. 17, 32, and 47. Their half-coating with $\mathrm{SiO} 2$, gold, nickel, or iron resulted in a Janus two-faced asymmetric structure of particles, with the last two coatings providing magnetic remote control for micro-/nanomotors. In the presence of fuel, micro-/nanomotors with diameters of 90-800 nm showed increased diffusion, indicating a chemophoretic propulsion mechanism. In terms of micro/nanomotor biocompatibility, those with immobilized GOx and UR were harmless to HeLa cells up to a concentration of $32 \mathrm{~g} \mathrm{~mL} 1$.

Submicron Janus micromotors $(800 \mathrm{~nm})$ were made using poly (dopamine) (PDA)-coated silica particles with one hemisphere pegylated with poly (1-lysine)-graft-poly (ethylene glycol) (PLL-g-PEG) for CAT and GOx immobilization utilizing a similar asymmetric structure idea. 48 Depending on the concentration of glucose as a primary fuel, the micromotors showed increased diffusion. It's worth mentioning that the scientists found that the diffusion coefficient in simulated biological media was greater than the diffusion coefficient in the buffer. This is in contrast to previous findings in the micromotor literature, which showed that the velocity of micromotors was reduced in complex biological media compared to buffers. In both phosphate-buffered saline (PBS) and aqueous solutions including urea as a fuel, 49 MSP-based core-shell nanobots $(340 \mathrm{~nm})$ with UR immobilized evenly on their surface showed increased diffusion. In the presence of urea, they were demonstrated to load and transport the drug doxorubicin to HeLa cells with enhanced release.16 Furthermore, anti-FGFR3 antibody-conjugated UR-powered MSPs (481 nm) for active self-propulsion in urine and targeting bladder cancer spheroids were created. 50 Passive MSPs without antibody achieved around 14 times greater internalization than actively propelled nanorobots with a targeting moiety. As a result of the nanorobots' generation of ammonia and the action of an anti-FGFR3 antibody inhibiting the fibroblast growth factor (FGF) signaling pathway, spheroid proliferation was reduced.

Covalent immobilization of lipase from Candida rugosa on the surface of MSP-based spheres $(430 \mathrm{~nm})$ was used to create hydrolase-powered nanomotors. Lipase has a dual function, generating energy by hydrolyzing water-soluble triacetin (glyceryl triacetate) as a fuel and degrading tributyrin, which is similar to blood lipid. 29

\subsection{Polymer-based systems}

Antibodies, peptides, and oligonucleotides are known to bind to streptavidin-functionalized polystyrene (PS) microspheres, which have been used in diagnostics and affinity-based purifications. 51 Biocompatible micromotors were made by immobilizing biotinylated CAT or UR evenly on the surface of streptavidin-functionalized PS microspheres (790 nm) based on the high streptavidin affinity for biotin. When the fuel concentration was raised, the micromotors showed greater diffusion (H2O2, urea). 7 
Another method for making enzyme-powered micro-/nanomotors is layer-by-layer (LbL) self-assembly of polymers into spherical forms with complicated connections. Polyelectrolyte multilayered Janus capsules $(8 \mathrm{~m})$ were half-coated with a gold layer for chemical conjugation of CAT and with nickel for magnetic remote control to create soft polymer-based hybrid micromotors with a light-triggered drug release mechanism. 52 At 0.1 percent $\mathrm{H} 2 \mathrm{O} 2$ and physiological temperature, the resultant micromotors were bubble propelled and followed a circular trajectory with a velocity of $4.2 \mathrm{~m} \mathrm{~s} 1$. Doxorubicin was loaded into anionic poly (styrene sulfonate) and cationic poly (allylamine hydrochloride) multilayers of micromotors and released on-demand by the NIR effect after being loaded into an ethanol solution.

The coupling of microfluidics with polymer hydrogel is regarded as a cutting-edge technological platform, with applications in cell culture, biosensing, sensing, and separation, the creation of improved microfluidic devices53, and the construction of artificial micro-/nanomotors. 54 Biocompatible hydrogel-based micromotors were created using a microfluidic chip and a bottom-up construction approach. Asymmetric motors $(20 \mathrm{~m})$ were made from cross-linked PEG diacrylate with entrapped CAT and dextran and bubble propelled at 2-4 percent $\mathrm{H} 2 \mathrm{O} 2$, usually in a circular trajectory. 55

\subsection{Metal-polymer hybrid systems}

The symmetric and asymmetric immobilization of CAT, HRP, and cytochrome c (Cyt c) on opposite ends of nanorods made of polypyrrole (PPy) and Au segments resulted in a series of hemeproteinase-driven nanomotors $(200 \mathrm{~nm} 1.5-2.5 \mathrm{~m})$. As a result of the enzymes being at different ends of the nanorods, gradients of reactants and products form along the nanorods, allowing self-propulsion via diffusiophoresis. 56 Electrophoresis was assumed for the self-propulsion mechanism of (HRP) PPy-Au (Cyt c) nanorods, since both enzymes were capable of transporting electrons to and from solid substrates. 37 In situ in the solution of nanomotors, XOD-catalyzed hypoxanthine turnover provided superoxide anion radicals and hydrogen peroxide.

\section{Supramolecular Assemblies Powered by Enzymes}

\subsection{Polymer stomatocytes}

Nanomotors $(400 \mathrm{~nm})$ with specific stomatocyte shapes were constructed using biocompatible supramolecular assemblies based on amphiphilic copolymers of PEG and PS. 57 The trapping of both CAT and a combination of CAT and GOx (1:3) in the "stoma" of stomatocytes resulted in nanomotors that were self-propelled in a solution of glucose $(5 \mathrm{~mm})$ by expelling oxygen molecules. It's worth mentioning that self-diffusiophoresis and bubble propulsion were found to govern the movement of similar platinum-based stomatocyte nanomotors in response to $\mathrm{H} 2 \mathrm{O} 2$ concentration. 58 
PEG-b-PS-based polymersomes were extruded over a membrane, resulting in their reassembly into $150 \mathrm{~nm}$-sized polymersomes, which were tiny enough for internalization by the cells. The generated osmotic pressure shock 59 resulted in a stomatocyte form with encapsulated CAT with the addition of PEG as a fusogen of the polymersome membrane. 60 HeLa cells were able to pick up the enzyme-driven stomatocyte nanomotors and penetrate a monolayer of pulmonary artery endothelial cells. Biodegradable PEG-b-PDLLA-based stomatocytes were created by replacing the PS block with poly (d, l-lactide) (PDLLA). Covalent bonding of enzymatic biomolecules and surface functionalization of the resultant stomatocytes were made possible by using PEG ends with azide groups. Other functionalized molecules were connected to modified groups on the stomatocytes' outer surface, whilst CAT and GOx were covalently bonded inside the stoma of the stomatocytes. 61

\subsection{MOFs}

Two-dimensional MOFs are an interesting material platform for immobilizing molecular catalysts due to their large pore volume and specific surface area. 62 Kong et al. recently developed pH-responsive, CAT-powered micromotors by enclosing CAT and succinylated-lactoglobulin in a zeolitic imidazolate framework-L. (ZIF-L). 63 The self-propulsion of the CAT-immobilized porous framework particles with a cruciate flowerlike shape was dependent on the $\mathrm{H} 2 \mathrm{O} 2$ concentration at $\mathrm{pH}$ 7. In an acidic environment (pH 5), succinylated-lactoglobulin experiences a sol-gel transition, preventing $\mathrm{H} 2 \mathrm{O} 2$ access and resulting in random Brownian motion in the micromotors.

\section{Conclusion}

Because of their biocompatibility, bioavailability, and capacity to convert chemical energy into mechanical work even in ionic environments, enzyme-powered micro/nanomotors have attracted a lot of interest in the field of hybrid micro/nanomotors. Active targeting, cargo loading and release, and biosensing have all been made possible because of their sophisticated architecture and functionalization. A thorough knowledge of structural complimentary domains and synergistic interactions will give unique features for the bottom-up self-assembly of numerous enzymes in the development of bioinspired biomolecular machines in future concepts.

A concentration gradient will control the movement of enzyme-powered nanorobots in a certain direction. They will travel in medical applications in response to a gradient of chemicals released at areas of tissue injury or inflammation. Wilson et al. recently published a paper on CAT-powered biodegradable poly (lactic-co-glycolic acid) micromotors that moved in response to a hydrogen peroxide concentration gradient for the administration of a periodontal disease medication. 64 A phorbol ester activated macrophage cell was used to study the chemotactic self-propulsion of micromotors in an in vitro model of inflammatory periodontitis. 
References

1R. Mallik, S. P. Gross, Curr. Biol. 2004, 14, R971- R982.

2X. Ma, A. C. Hortelão, T. Patiño, S. Sánchez, ACS Nano 2016, 10, 9111- 9122.

3

3aC. Riedel, R. Gabizon, C. A. M. Wilson, K. Hamadani, K. Tsekouras, S. Marqusee, S. Pressé, C. Bustamante, Nature 2015, 517, 227;

3bR. Golestanian, Phys. Rev. Lett. 2015, 115, 108102.

4X. Zhao, K. Gentile, F. Mohajerani, A. Sen, Acc. Chem. Res. 2018, 51, 2373- 2381.

5A.-Y. Jee, S. Dutta, Y.-K. Cho, T. Tlusty, S. Granick, Proc. Natl. Acad. Sci. USA 2018, 115, $14-18$.

6S. Sengupta, K. K. Dey, H. S. Muddana, T. Tabouillot, M. E. Ibele, P. J. Butler, A. Sen, J. Am. Chem. Soc. 2013, 135, 1406- 1414.

7K. K. Dey, X. Zhao, B. M. Tansi, W. J. Méndez-Ortiz, U. M. Córdova-Figueroa, R. Golestanian, A. Sen, Nano Lett. 2015, 15, 8311-8315.

8S. Sanchez, A. A. Solovev, Y. Mei, O. G. Schmidt, J. Am. Chem. Soc. 2010, 132.

9J. Orozco, V. García-Gradilla, M. D'Agostino, W. Gao, A. Cortés, J. Wang, ACS Nano 2013, $7,818-824$.

10W. Waldhäusl, G. Kleinberger, A. Korn, R. Dudczak, P. Bratusch-Marrain, P. Nowotny, Diabetes 1979, 28, 577-584.

11M. Yamaguchi, M. Mitsumori, Y. Kano, Proceedings of the 20th Annual International Conference of the IEEE Engineering in Medicine and Biology Society, Vol. 20, Biomedical Engineering Towards the Year 2000 and Beyond (Cat. No.98CH36286) 1998, pp. 1763-1766.

12N. Mano, A. Heller, J. Am. Chem. Soc. 2005, 127, 11574- 11575.

13D. Pantarotto, W. R. Browne, B. L. Feringa, Chem. Commun. 2008, 1533- 1535. 
14J. Kopstein, O. M. Wrong, Clin. Sci. 1977, 52, 9- 17.

15L. Magos, J. Appl. Toxicol. 1987, 7, 413.

16A. C. Hortelão, T. Patiño, A. Perez-Jiménez, À. Blanco, S. Sánchez, Adv. Funct. Mater. 2018, 28, 1705086.

17T. Patino, A. Porchetta, A. Jannasch, A. Lladó, T. Stumpp, E. Schäffer, F. Ricci, S. Sánchez, Nano Lett. 2019, 19, 3440-3447.

18S. Sattayasamitsathit, K. Kaufmann, M. Galarnyk, R. Vazquez-Duhalt, J. Wang, RSC Adv. 2014, 4, 27565-27570.

19Z. Wu, X. Lin, X. Zou, J. Sun, Q. He, ACS Appl. Mater. Interfaces 2015, 7, 250- 255.

20V. V. Singh, K. Kaufmann, B. Esteban-Fernández de Ávila, M. Uygun, J. Wang, Chem. Commun. 2016, 52, 3360-3363.

21X. Ma, A. C. Hortelao, A. Miguel-López, S. Sánchez, J. Am. Chem. Soc. 2016, 138, $13782-13785$.

22

22aP. Pinyou, V. Blay, L. M. Muresan, T. Noguer, Mater. Horiz. 2019, 6, 1336- 1358;

22bH. H. Nguyen, S. H. Lee, U. J. Lee, C. D. Fermin, M. Kim, Materials 2019, 12, 121 ;

22cS. Ferri, K. Kojima, K. Sode, J. Diab. Sci. Technol. 2011, 5, 1068- 1076.

23J. Kaushal, S. Mehandia, G. Singh, A. Raina, S. K. Arya, Biocatal. Agricult. Biotechnol. 2018, 16, 192- 199.

24A. N. P. Hiner, J. Hernández-Ruiz, G. A. Williams, M. B. Arnao, F. García-Cánovas, M. Acosta, Arch. Biochem. Biophys. 2001, 392, 295- 302.

25

25aH. Y. Chung, B. S. Baek, S. H. Song, M. S. Kim, J. I. Huh, K. H. Shim, K. W. Kim, K. H. Lee, Age 1997, 20, 127-140; 
25bE. E. Kelley, N. K. H. Khoo, N. J. Hundley, U. Z. Malik, B. A. Freeman, M. M. Tarpey, Free Rad. Biol. Med. 2010, 48, 493- 498.

26

26aA. Commault, R. Weld, Whole-cell Biosensors for Monitoring Bioremediation in Biofilms in Bioremediation: Current Research and Emerging Technologies (Ed.: ). Caister Academic Press, Norfolk, 2016, pp. 75- 92;

26bR. Ramesh, P. Selvan, J. Kumar, M. Gowthaman, S. D'Souza, N. Kamini, Mater. Sci. Engin. C 2015, 49, 786- 792.

27C. R. Carlini, R. Ligabue-Braun, Toxicon 2016, 110, 90- 109.

28M. Luo, S. Li, J. Wan, C. Yang, B. Chen, J. Guan, Langmuir 2020, 10.1021/acs.langmuir.9b03315.

29L. Wang, A. C. Hortelão, X. Huang, S. Sánchez, Angew. Chem. Int. Ed. 2019, 58, 79927996;

Angew. Chem. 2019, 131, 8076-8080.

30P. S. Schattling, M. A. Ramos-Docampo, V. Salgueiriño, B. Städler, ACS Nano 2017, 11, 3973- 3983.

31T. Patiño, N. Feiner-Gracia, X. Arqué, A. Miguel-López, A. Jannasch, T. Stumpp, E. Schäffer, L. Albertazzi, S. Sánchez, J. Am. Chem. Soc. 2018, 140, 7896- 7903.

32X. Ma, A. Jannasch, U.-R. Albrecht, K. Hahn, A. Miguel-López, E. Schäffer, S. Sánchez, Nano Lett. 2015, 15, 7043-7050.

33X. Arqué, A. Romero-Rivera, F. Feixas, T. Patiño, S. Osuna, S. Sánchez, Nat. Commun. 2019, 10, 2826.

34T. Patiño, X. Arqué, R. Mestre, L. Palacios, S. Sánchez, Acc. Chem. Res. 2018, 51, 26622671.

35

35aS. Gáspár, Nanoscale 2014, 6, 7757-7763; 
35bL. Sonntag, J. Simmchen, V. Magdanz, Molecules 2019, 24, 3410.

36R. Golestanian, Phys. Rev. Lett. 2009, 102, 188305.

37I.-A. Pavel, A.-I. Bunea, S. David, S. Gáspár, ChemCatChem 2014, 6, 866- 872.

38

38aK. K. Dey, A. Sen, J. Am. Chem. Soc. 2017, 139, 7666-7676;

38bF. Mohajerani, X. Zhao, A. Somasundar, D. Velegol, A. Sen, Biochemistry 2018, 57, $6256-6263$.

39A. S. Mikhailov, R. Kapral, Proc. Natl. Acad. Sci. USA 2015, 112, E3639- E3644.

40A. Somasundar, S. Ghosh, F. Mohajerani, L. N. Massenburg, T. Yang, P. S. Cremer, D. Velegol, A. Sen, Nat. Nanotechnol. 2019, 14, 1129- 1134.

41J. Agudo-Canalejo, P. Illien, R. Golestanian, Nano Lett. 2018, 18, 2711- 2717.

42

42aY. Mei, G. Huang, A. A. Solovev, E. B. Ureña, I. Mönch, F. Ding, T. Reindl, R. K. Y. Fu, P. K. Chu, O. G. Schmidt, Adv. Mater. 2008, 20, 4085- 4090;

42bA. A. Solovev, Y. Mei, E. Bermúdez Ureña, G. Huang, O. G. Schmidt, Small 2009, 5, $1688-1692$;

42cQ. Chi, Z. Wang, F. Tian, J. A. You, S. Xu, Micromachines 2018, 9, 537.

43P. Qiu, B. Ma, C.-T. Hung, W. Li, D. Zhao, Acc. Chem. Res. 2019, 52, 2928- 2938.

44Y. Zhou, G. Quan, Q. Wu, X. Zhang, B. Niu, B. Wu, Y. Huang, X. Pan, C. Wu, Acta Pharm. Sin. B 2018, 8, 165- 177.

45J. Simmchen, A. Baeza, D. Ruiz, M. J. Esplandiu, M. Vallet-Regí, Small 2012, 8, $2053-$ 2059.

46X. Ma, S. Sanchez, Chem. Commun. 2015, 51, 5467- 5470. 
47aX. Ma, X. Wang, K. Hahn, S. Sánchez, ACS Nano 2016, 10, 3597-3605;

47bX. Ma, S. Sánchez, Tetrahedron 2017, 73, 4883- 4886.

48P. Schattling, B. Thingholm, B. Städler, Chem. Mater. 2015, 27, 7412- 7418.

49

49aL. Soler, C. Martínez-Cisneros, A. Swiersy, S. Sánchez, O. G. Schmidt, Lab Chip 2013, 13, 4299- 4303;

49bV. Garcia-Gradilla, J. Orozco, S. Sattayasamitsathit, F. Soto, F. Kuralay, A. Pourazary, A. Katzenberg, W. Gao, Y. Shen, J. Wang, ACS Nano 2013, 7, 9232- 9240.

50A. C. Hortelão, R. Carrascosa, N. Murillo-Cremaes, T. Patiño, S. Sánchez, ACS Nano 2019, 13, 429- 439.

51J. N. Wilson, Y. Wang, J. J. Lavigne, U. H. F. Bunz, Chem. Commun. 2003, 1626- 1627.

52Y. Wu, X. Lin, Z. Wu, H. Möhwald, Q. He, ACS Appl. Mater. Interfaces 2014, 6, 1047610481.

53C. B. Goy, R. E. Chaile, R. E. Madrid, React. Funct. Polymers 2019, 145, 104314.

54Y. Yu, J. Guo, M. Zou, L. Cai, Y. Zhao, Chem. Asian J. 2019, 14, 2417- 2430.

55S. Keller, S. P. Teora, G. X. Hu, M. Nijemeisland, D. A. Wilson, Angew. Chem. Int. Ed. 2018, 57, 9814- 9817;

Angew. Chem. 2018, 130, 9962- 9965.

56A.-I. Bunea, I.-A. Pavel, S. David, S. Gáspár, Chem. Commun. 2013, 49, 8803- 8805.

57

57aL. K. E. A. Abdelmohsen, M. Nijemeisland, G. M. Pawar, G.-J. A. Janssen, R. J. M. Nolte, J. C. M. van Hest, D. A. Wilson, ACS Nano 2016, 10, 2652-2660; 
57bM. Nijemeisland, L. K. E. A. Abdelmohsen, W. T. S. Huck, D. A. Wilson, J. C. M. van Hest, ACS Cent. Sci. 2016, 2, 843-849.

58D. A. Wilson, B. de Nijs, A. van Blaaderen, R. J. M. Nolte, J. C. M. van Hest, Nanoscale 2013, 5, 1315- 1318 .

59Y. Men, W. Li, G.-J. Janssen, R. S. M. Rikken, D. A. Wilson, Nano Lett. 2018, 18, 20812085.

60J. Sun, M. Mathesh, W. Li, D. A. Wilson, ACS Nano 2019, 13, 10191- 10200.

61B. J. Toebes, F. Cao, D. A. Wilson, Nat. Commun. 2019, 10, 5308.

62M. Safaei, M. M. Foroughi, N. Ebrahimpoor, S. Jahani, A. Omidi, M. Khatami, Trends Anal. Chem. 2019, 118, 401- 425.

63S. Gao, J. Hou, J. Zeng, J. J. Richardson, Z. Gu, X. Gao, D. Li, M. Gao, D.-W. Wang, P. Chen, V. Chen, K. Liang, D. Zhao, B. Kong, Adv. Funct. Mater. 2019, 29, 1808900.

64J. Wang, B. J. Toebes, A. S. Plachokova, Q. Liu, D. Deng, J. A. Jansen, F. Yang, D. A. Wilson, Adv. Healthcare Mater. 2020, 9, 1901710.

65. Abdelhamid, H. N., M. Dowaidar, M. Hällbrink, and Ü. Langel. 2019. "Cell Penetrating Peptides-Hierarchical Porous Zeolitic Imidazolate Frameworks Nanoparticles: An Efficient Gene Delivery Platform." SSRN Electron. J. https://papers.ssrn.com/sol3/papers.cfm?abstract_id=3435895.

66. Abdelhamid, Hani Nasser, Moataz Dowaidar, Mattias Hällbrink, and Ülo Langel. 2020. "Gene Delivery Using Cell Penetrating Peptides-Zeolitic Imidazolate Frameworks." Microporous and Mesoporous Materials: The Official Journal of the International Zeolite Association $300 \quad$ (June): 110173. https://doi.org/10.1016/j.micromeso.2020.110173.

67. Abdelhamid, Hani Nasser, Moataz Dowaidar, and Ülo Langel. 2020. "Carbonized Chitosan Encapsulated Hierarchical Porous Zeolitic Imidazolate Frameworks Nanoparticles for Gene Delivery." Microporous and Mesoporous Materials: The Official Journal of the International Zeolite Association 302 (August): 110200. https://doi.org/10.1016/j.micromeso.2020.110200.

68. Ahmad, Almeman, Khalaf Hassan, Rasool Semaab, Moataz Dowaidar, and Al Orainy Mohammad. 2013. "The Impact of CYP2C19 Polymorphism on Platelet Reactivity for Guiding Clopidogrel Treatment and Cost Analysis." Journal of the Saudi Heart Association 25 (2): 107. https://doi.org/10.1016/j.jsha.2013.03.005. 
69. Algahsham, Abdullah, Ahmad A. A. Settin, Ahmad Ali, and Hisham Ismail. n.d. "Association of MTHFR C677T and A1298C Polymorphisms with Hypertension among Saudi Subjects from Qassim Region." International Journal of Health $\begin{array}{llllll}\text { Sciences } & 6 & \text { (1). } & \text { Accessed }\end{array}$ http://ijhs.org.sa/index.php/journal/article/view/312.

70. Algasham, Abdullah, Hisham Ismail, Moataz Dowaidar, and Ahmad A. Settin. 2011. "Methylenetetrahydrofolate Reductase (MTHFR) and Angiotensin Converting Enzyme (ACE) Gene Polymorphisms among Saudi Population from Qassim Region.” International Journal of Health Sciences 5 (2 Suppl 1): 3-4. https://www.ncbi.nlm.nih.gov/pubmed/23284552.

71. Alghasham, Abdullah, Ahmad Ali, Hisham Ismail, Moataz Dowaidar, and Ahmad A. Settin. 2012. "CYP2J2 -50 G/T and ADRB2 G46A Gene Polymorphisms in Saudi Subjects with Hypertension." Genetic Testing and Molecular Biomarkers 16 (9): 1027-31. https://doi.org/10.1089/gtmb.2012.0006.

72. Alghasham, Abdullah, Ahmad A. Settin, Ahmad Ali, Moataz Dowaidar, and Hisham Ismail. 2012a. "Association of MTHFR C677T and A1298C Gene Polymorphisms with Hypertension." International Journal of Health Sciences 6 (1): 3-11. https://doi.org/10.12816/0005968.

73. Moataz Dowaidar. 2012b. "Association of MTHFR C677T and A1298C Gene Polymorphisms with Hypertension.” International Journal of Health Sciences 6 (1): 3-11. https://doi.org/10.12816/0005968.

74. Ali, Ahmad, Abdullah Alghasham, Hisham Ismail, Moataz Dowaidar, and Ahmad Settin. 2013. "ACE I/D and eNOS E298D Gene Polymorphisms in Saudi Subjects with Hypertension." Journal of the Renin-Angiotensin-Aldosterone System: JRAAS 14 (4): 348-53. https://doi.org/10.1177/1470320312459976.

75. Ali, Ahmed A. A., Nahla M. Wassim, Moataz M. Dowaidar, and Ahmed E. Yaseen. 2013a. "Genetic Polymorphism of CYP2D6 Gene among Egyptian Hypertensive Cases." The Journal of Basic \& Applied Zoology 66 (4): 228-33. https://doi.org/10.1016/j.jobaz.2012.12.002.

76. Ali, Ahmed A. A., Nahla M. Wassim, Moataz Dowaidar, and Ahmed E. Yaseen. 2013b. "Association of eNOS (E298D) and CYP2J2 (-50G/T) Gene Polymorphisms with Hypertension among Egyptian Cases." The Journal of Basic \& Applied Zoology 66 (4): 234-41. https://doi.org/10.1016/j.jobaz.2012.12.001.

77. Moataz Dowaidar. 2013c. "Association of eNOS (E298D) and CYP2J2 (-50G/T) Gene Polymorphisms with Hypertension among Egyptian Cases." The Journal of Basic \& Applied Zoology 66 (4): 234-41. https://doi.org/10.1016/j.jobaz.2012.12.001.

78. Aljarallah, Badr, Ahmed Ali, Moataz Dowaidar, and Ahmad Settin. 2011. "Prevalence of $\alpha$-1-Antitrypsin Gene Mutations in Saudi Arabia." Saudi Journal of Gastroenterology: Official Journal of the Saudi Gastroenterology Association 17 (4): 256-60. https://doi.org/10.4103/1319-3767.82580.

79. Dowaidar, M., M. Gestin, C. P. Cerrato, and H. Margus. 2017. "Role of Autophagy in PepFect14 Transfection." https://www.diva-portal.org/smash/record.jsf?pid=diva2:1077716. 
80. Dowaidar, M., J. Regberg, D. A. Dobchev, and T. Lehto. 2017. "Refinement of a Quantitative Structure-activity Relationship Model for Prediction of Cell-Penetrating Peptide Based Transfection Systems." International Journal of. https://link.springer.com/content/pdf/10.1007/s10989-016-9542-8.pdf.

81. Dowaidar, Moataz. 2017. "In-Silico Design of Peptide-Based Transfection Systems, in-Vitro Validation, and up-Take Pathways Investigation." Department of Neurochemistry, Stockholm University.

82. Moataz Dowaidar. 2018. "Chimeric Gene Delivery Vectors : Design, Synthesis, and Mechanisms from Transcriptomics Analysis." Department of Biochemistry and Biophysics, Stockholm University. https://www.diva-portal.org/smash/record.jsf?pid=diva2:1242000.

83. Moataz Dowaidar. 2021a. "Cardiometabolic Conditions Could Be Related to Vitamin D Deficiency. The Genetic Determinants That Affect Vitamin D Pathways May Be Solved with Nanomedicines." https://doi.org/10.31219/osf.io/nqewr.

84. Moataz Dowaidar. 2021b. "Different Insulin Resistance and Inflammation Pathways Are Influenced by Genetic Factors in Metabolic Syndrome. Gene Therapy Enables Early Recognition and Treatment of the Genetic Factors." https://doi.org/10.31219/osf.io/gqwj2.

85. Moataz Dowaidar. 2021c. "Gene Therapy Has Been Shown to Be Valuable for Understanding Complex Disease Pathophysiologies. The Medical Profession as a Whole Will Have to Invest in Specialized Investigations." https://doi.org/10.31219/osf.io/8fg9y.

86. Moataz Dowaidar. 2021d. "Genetic and Epigenetic Discoveries Hold Promising Avenues in Cardiovascular Prevention and Management (CVDs). Key Nucleic Acids Are Being Researched and Developed for Medicinal Use." https://doi.org/10.31219/osf.io/hk7pe.

87. Moataz Dowaidar. 2021e. "Genome Editing Can Now Be Carried out in an Isogenic Setting. It Can Be Effectively Transmitted to Somatic Tissues in Mice, but Not to Humans. Despite These Doubts, CRIS Has Great Potential as a Medical Promise." https://doi.org/10.31219/osf.io/4rn3v.

88. Moataz Dowaidar. 2021f. "Genome-Wide Association Experiments Have Uncovered a Slew of Cardiometabolic Trait-Associated Variants. This Information Can Be Useful in the Implementation of New Diagnostic and Treatment Strategies." https://doi.org/10.31219/osf.io/4vws8.

89. Moataz Dowaidar. 2021g. "Genome-Wide Association Studies (GWAS) Have Revolutionized Our View of Human Health and Disease Genetics and Offered Novel Gene Therapy Targets." https://doi.org/10.31219/osf.io/rvm3z.

90. Moataz Dowaidar. 2021h. "Metabolic Syndrome_the Presence of Inflammatory Mechanisms in Abdominal Obesity Is Undeniable, Gene Therapy Using Nanoparticles and Adenoviruses Technologies Is Promising." https://doi.org/10.31219/osf.io/2j5xt. 
91. Moataz Dowaidar. 2021i. "miRNAs May Be Used as Preventive Agents for Metabolic Diseases in the near Future. Understanding the Interplay between pro-Adipogenic and Anti-Ad Pipogenic miRNA' Could Lead to New Biomarkers." https://doi.org/10.31219/osf.io/3dr8c.

92. Moataz Dowaidar. 2021j. "Nanomedicine Has Elegantly Attempted to Cure Multiple Gene Polymorphisms and Mutations in Cardiovascular Diseases Using Gene Therapy Techniques." https://doi.org/10.31219/osf.io/d3x8g.

93. Moataz Dowaidar. 2021k. "Thrombosis Pathways and Therapeutic Strategies." https://doi.org/10.31219/osf.io/57vyz.

94. Moataz Dowaidar. 20211. "What Genomic Research Has Told Us about the Obesity and Its Possible Gene Therapy Targets." https://doi.org/10.31219/osf.io/ym49s.

95. Moataz Dowaidar. 2021m. "Exosomes Can Make the Use of Circulating miRNA as a Biomarker More Feasible. The Aim of Gene Therapy Should Be to Learn Everything There Is to Know about miRNA Activity." https://oi.org/10.31219/osf.io/edkua.

96. Moataz Dowaidar. 2021n. "Anti-Sense Pathways Have Been Generated Using siRNA. The Liver and Other Often Used Organs Will Now Be Targeted." https://doi.org/10.31219/osf.io/m6xvp.

97. Moataz Dowaidar. 20210. "CrisPR/CRIS Systems Are Highly Effective and Useful for Genomic Manipulation. Despite This, Cardiac Treatment Remains Difficult due to Existing Genome Editing and Delivery Processes.” https://doi.org/10.31219/osf.io/3nwzd.

98. Moataz Dowaidar. 2021p. "Discoveries in Gene-Environment Interactions That Influence CVD, Lipid Traits, Obesity, Diabetes, and Hypertension Appear to Be Able to Influence Gene Therapy.” https://doi.org/10.31219/osf.io/cr5af.

99. Moataz Dowaidar. 2021q. "Genome Editing's Potential Target Diseases in the Cardiovascular Field." https://doi.org/10.31219/osf.io/gc23p.

100. Moataz Dowaidar. 2021r. "Key Genetic Factors in the Metabolic Syndrome Predisposition Which May Be a Therapeutic Options by Gene Therapy." https://doi.org/10.31219/osf.io/f38sk.

101. Moataz Dowaidar. 2021s. "miRNA Can Be a Part of Both the Onset and Cure of Coronary Heart Disease.” https://doi.org/10.31219/osf.io/teqh8.

102. Moataz Dowaidar. 2021t. "Preclinical Studies and Clinical Trials Have Sparked Interest in Certain Biological Medications for Atherosclerotic Coronary Heart Disease." https://doi.org/10.31219/osf.io/ts8mh.

103. Moataz Dowaidar. 2021u. "Researchers Would Be Able to Develop a Detailed Picture of Chromatin in Disease, Which Would Be Useful for Gene Therapy." https://doi.org/10.31219/osf.io/m9z48.

104. Moataz Dowaidar. 2021v. "The Cardiometabolic-Based Chronic Disease Model Lays the Foundations for Accurate, Evidence-Based Preventive Targeting and Gene Therapy." https://doi.org/10.31219/osf.io/up9z4.

105. Moataz Dowaidar. 2021w. "2D MOFs Have Unique Features for Biological Applications. They Can Be Utilized for Gene Therapy, Bioimaging, Biosensing, Photodynamic Therapy, and Tissue Engineering." https://doi.org/10.31219/osf.io/4q9ct. 
106. Moataz Dowaidar. 2021x. "3D Bioprinting for Enhanced Vascularization, and Gene Editing to Provide a More Favorable Immunological Response Are Just Some of the Potential Uses of Carbon Materials." https://doi.org/10.31219/osf.io/v2xy8.

107. Moataz Dowaidar. 2021y. "Anderson-Fabry Disease Can Be a Target for Gene Therapy." https://doi.org/10.31219/osf.io/tcgka.

108. Moataz Dowaidar. 2021z. "Antisense Oligonucleotides (ASOs) and CRISPR Systems Are Promising Gene Therapy Treatments for Alzheimer's Disease." https://doi.org/10.31219/osf.io/ws796.

109. Moataz Dowaidar. 2021aa. "Any Alteration in PPAR Genomic Sequence, Splicing Pattern, or PTM Is Likely to Cause Major Alterations in Its Function. In Personalized Medicine, Such Data Becomes More Significant in Gene Therapy Design." https://doi.org/10.31219/osf.io/y8n79.

110. Moataz Dowaidar. 2021ab. "Applying Genome-Wide Association Technology to Brain Diseases Enables the Discovery of lncRNas Targets for Gene Therapy." https://doi.org/10.31219/osf.io/hm4eu.

111. Moataz Dowaidar. 2021ac. "Autophagy and Proteostasis Adjustment Role in Normal Brain Function and Neurodegenerative Disorders.” https://doi.org/10.31219/osf.io/m4yra.

112. Moataz Dowaidar. 2021ad. "Basal Ganglia-Cerebellar and Brainstem-Cerebellar Circuits May Interact Improperly with Dystonia. Linking Network Disruptions to Cell Failure Will Enable Understanding Pathophysiology and Designing Gene Therapy Methods." https://doi.org/10.31219/osf.io/8w35s.

113. Moataz Dowaidar. 2021ae. "Blood Products Are Used to Treat a Multitude of Diseases, so the Blood Transfusion System Needs to Be Enhanced. CRISPR/Cas9 Has Made It Viable to Make HLA Class I-Deleted Blood Products to Avoid Rejection." https://doi.org/10.31219/osf.io/egr3n.

114. Moataz Dowaidar. 2021af. "Calixarenes (CAs) Are Promising in Biomedicine, Biosensing, Bioimaging and Gene Delivery Systems." https://doi.org/10.31219/osf.io/n9vjy.

115. Moataz Dowaidar. 2021ag. "CAR T Cell Research Has Quickly Advanced from the Bench to the Clinic and Back. The Results of the Trials Have Revealed New Mechanisms." https://doi.org/10.31219/osf.io/f9wm7.

116. Moataz Dowaidar. 2021ah. "CAR T-Cell Treatment Remains Clinically Challenging. Therapeutic Strategies May Be Designed to Cut off Immunotherapy Utilizing Safety Switches." https://doi.org/10.31219/osf.io/s7x4y.

117. Moataz Dowaidar. 2021ai. "Central Nervous System Gene Therapy Has Entered a New Development Paradigm. New Techniques Are Being Employed for a Wide Range of Illness Indications and Pathways." https://doi.org/10.31219/osf.io/j49wz.

118. Moataz Dowaidar. 2021aj. "Chronic Obstructive Pulmonary Condition (COPD) Is a Prevalent, Preventable, and Curable Illness with Persistent Respiratory Symptoms and Airflow Limitation.” https://doi.org/10.31219/osf.io/vkdut.

119. Moataz Dowaidar. 2021ak. "CircRNAs Have the Potential to Aid in the Diagnosis and Treatment of Lipid Diseases.” https://doi.org/10.31219/osf.io/y3hp4. 
120. Moataz Dowaidar. 2021al. "Clinical Symptoms, Underlying Pathogenesis, and the Prospect of Tailored Therapies Have All Benefited from Genetic Discoveries in Parkinson's Disease." https://doi.org/10.31219/osf.io/pdzqb.

121. Moataz Dowaidar. 2021am. "Code Distribution of siRNA for Cancer Genes such as p53 and Bcl2 Family Genes Has Demonstrated Efficacy in Killing Cancer Cells. Nanoparticles Can Produce a Surface Where Numerous Drugs May Be Coupled, Allowing Combinatory Treatment." https://doi.org/10.31219/osf.io/hvcse.

122. Moataz Dowaidar. 2021an. "Cognitive Deficiencies Pathophysiology Are Mainly an Unknown Area. Curing the Neurological Conditions Could Be an Objective for Gene Therapy." https://doi.org/10.31219/osf.io/23xf8.

123. Moataz Dowaidar. 2021ao. "CRISPR-Based Gene Editing Is Presently Being Tried in Many Clinical Trials.” https://doi.org/10.31219/osf.io/qbngx.

124. Moataz Dowaidar. 2021ap. "CRISPR-Cas9 Gene Editing as a Tool for Developing Immunotherapy for Cancer.” https://doi.org/10.31219/osf.io/dvr4t.

125. Moataz Dowaidar. 2021aq. "CRISPR/Cas System Research Has Advanced Significantly in Biological sciences.There Are Still Many Challenges to Effective Delivery before Efficient Gene Editing May Be Achieved." https://doi.org/10.31219/osf.io/mc26v.

126. Moataz Dowaidar. 2021ar. "CRISPR/Cas9 Genome Editing Technology Applications in Biological and Biomedical Fields." https://doi.org/10.31219/osf.io/ctqbe.

127. Moataz Dowaidar. 2021as. "Critical Limb Ischemia Potential Gene Therapy Strategies." https://doi.org/10.31219/osf.io/aqcpt.

128. Moataz Dowaidar. 2021at. "Deep Learning Algorithms for scRNAseq Analysis Have Yielded Positive Results, but There Are Still More Promising Ways That Need to Be Developed for Regenerative Medicine.” https://doi.org/10.31219/osf.io/dh2pt.

129. Moataz Dowaidar. 2021au. "Depression May Be Epigenetically Controlled by miRNAs Making It a Diagnostic or Gene Therapy Target." https://doi.org/10.31219/osf.io/fw65m.

130. Moataz Dowaidar. 2021av. "Dermatophytes: Role of Host Genetics in the Development of Illness." https://doi.org/10.31219/osf.io/mf3bu.

131. Moataz Dowaidar. 2021aw. "Developments in Biomedical Technology Will Increase the Importance of mRNA in Treating Brain Tumors, as Well as Other Malignancies." https://doi.org/10.31219/osf.io/tvj5x.

132. Moataz Dowaidar. 2021ax. "Downstream Processing of Virus, Virus-like Particles and Nanoparticulate Inclusion Bodies to Be Used as Gene Delivery Vehicles for Human Gene Therapy Applications." https://doi.org/10.31219/osf.io/exa3q.

133. Moataz Dowaidar. 2021ay. "Dravet Syndrome Is a Severe Developmental and Epileptic Encephalopathy. Fenfluramine and Gene Therapy Are Promising." https://doi.org/10.31219/osf.io/zvq8y.

134. Moataz Dowaidar. 2021az. "Exosomes' Function in Cardiovascular Protection and Neovascularization Implies That They Might Be Used to Treat Ischemia and Atherosclerotic Cardiovascular Diseases.” https://doi.org/10.31219/osf.io/2h8c7. 
135. Moataz Dowaidar. 2021ba. "Ferropsis Cell Death Can Cause Complications That May Be Difficult to Detect and Quantify: Autophagy Role and Possible Therapeutics." https://doi.org/10.31219/osf.io/zd2jg.

136. Moataz Dowaidar. 2021bb. "Following the Discovery of Anti-MDA5 Ab, the Clinical Understanding of Dermatomyositis Has Been Improved." https://doi.org/10.31219/osf.io/j2t5f.

137. Moataz Dowaidar. 2021bc. "For the Treatment of Cystic Fibrosis, RNA Medicines, Gene Transfer Therapies, and Gene Editing Treatments Have Potential." https://doi.org/10.31219/osf.io/6afzm.

138. Moataz Dowaidar. 2021bd. "Frontotemporal Dementia Is a Complex Disorder with a Wide Spectrum of Clinical Symptoms. Personalized Medicine and Gene Therapy Are Promising Strategies for Treatment." https://doi.org/10.31219/osf.io/gh4x7.

139. Moataz Dowaidar. 2021be. "G6PD Deficiency Is a Common Genetic Trait That Can Protect Heterozygotes from Dying from Malaria." https://doi.org/10.31219/osf.io/g2kza.

140. Moataz Dowaidar. 2021bf. "Gastric Cancer Is the World's Second-Largest Death Cause. Peptides Can Be Used to Deliver Radiation or Other Fatal Chemicals to Tumors." https://doi.org/10.31219/osf.io/eu5mj.

141. Moataz Dowaidar. 2021bg. "Gene Doping May Be Possible for Lifestyle Enhancement.” https://doi.org/10.31219/osf.io/8xkm5.

142. Moataz Dowaidar. 2021bh. "Gene Expression Assays Gather Evidence That They Can Provide Useful Therapeutic Information in Young Women." https://doi.org/10.31219/osf.io/d372s.

143. Moataz Dowaidar. 2021bi. "Gene Therapy and Genome-Editing Treatments That Can Protect Patients from Coronary Artery Disease Are under Investigation." https://doi.org/10.31219/osf.io/xqgf8.

144. Moataz Dowaidar. 2021bj. "Gene Therapy Approaches for Hemophilia A and B." https://doi.org/10.31219/osf.io/ufc4g.

145. Moataz Dowaidar. 2021bk. "Gene Therapy for the Central Nervous System Has Been Initiated. This Expansion Will Require Some Degree of Simplicity in Delivery Processes." https://doi.org/10.31219/osf.io/hdy5q.

146. Moataz Dowaidar. 2021bl. "Gene Therapy for the Treatment of Spinal Muscular Atrophy.” https://doi.org/10.31219/osf.io/kpz5f.

147. Moataz Dowaidar. 2021bm. "Gene Therapy May Benefit Inherited Ichthyoses with Concurrent Fungal Infections and Severe Ich Thyroidoses." https://doi.org/10.31219/osf.io/zxmun.

148. Moataz Dowaidar. 2021bn. "Gene Therapy May Target APOE for Alzheimer's Disease.” https://doi.org/10.31219/osf.io/3y52k.

149. Moataz Dowaidar. 2021bo. "Gene Therapy Promises Accurate, Targeted Administration and Overcoming Drug Resistance in Diverse Cancer Cells." https://doi.org/10.31219/osf.io/j34n6.

150. Moataz Dowaidar. 2021bp. "Gene Therapy Targeting FVIII, FIX for Haemophilia Treatment.” https://doi.org/10.31219/osf.io/qcbwp. 
151. Moataz Dowaidar. 2021bq. "Gene Therapy Targeting PRMT5 May Be Useful in Immunotherapy." https://doi.org/10.31219/osf.io/gkw8j.

152. Moataz Dowaidar. 2021br. "Gene Therapy Using Extracellular Vesicles Loaded with miRNA Derived from Bone Marrow Mesenchymal Stem Cells Is a Cell-Free Medication Delivery Method Used in a Variety of Diseases." https://doi.org/10.31219/osf.io/3znvw.

153. Moataz Dowaidar. 2021bs. "Genetic Engineered MSCs Are Attractive Possibilities for Regenerative Stem-Cell Therapy to Treat Several Liver Diseases." https://doi.org/10.31219/osf.io/4cfrd.

154. Moataz Dowaidar. 2021bt. "Genetic Variants Shared between Alzheimer's Disease and Parkinson's Disease Have Been Discovered in Blood and Brain Samples. Somatic Mosaicism Might Function as an Accelerator." https://doi.org/10.31219/osf.io/tr58n.

155. Moataz Dowaidar. 2021bu. "Genome-Wide Association Studies Promise to Discover Novel Indicators of Hypertension. Endothelin-Related SNPs Are Currently in Clinical Trials." https://doi.org/10.31219/osf.io/2n4wa.

156. Moataz Dowaidar. 2021bv. "Gingival and Intraventricular Haemorrhages Are Severe Newborn Diseases Causing Damage to White Matter and Neurological Dysfunction in Surviving Newborns Who Can Benefit from Gene Therapy." https://doi.org/10.31219/osf.io/qb84p.

157. Moataz Dowaidar. 2021bw. "Glioblastoma Therapeutic Approaches Were Established Utilizing Contemporary Discoveries in Delivering Medicines to the Brain as Smart Nanoparticles for Focused Therapy." https://doi.org/10.31219/osf.io/db4f6.

158. Moataz Dowaidar. 2021bx. "Haemophilia Gene Therapy Is in Clinical Studies, Making Continuous Safety and Efficacy Testing a Key Emphasis." https://doi.org/10.31219/osf.io/sa8ny.

159. Moataz Dowaidar. 2021by. "Hematopoietic Stem Cell Transplantation and Gene Therapy Are the Sole Treatments for Sickle Cell Disease and Other Hemoglobinopathies." https://doi.org/10.31219/osf.io/v8xqc.

160. Moataz Dowaidar. 2021bz. "Huntington's Disease Gene Therapy and Nanomedicines May Be Available Shortly." https://doi.org/10.31219/osf.io/rxvgd.

161. Moataz Dowaidar. 2021ca. "Hybrid Gene Therapy Designed to Fully Understand the Underlying Molecular Cancer Process May Be a Feasible Option." https://doi.org/10.31219/osf.io/ajyfd.

162. Moataz Dowaidar. 2021cb. "Hydrogels Are Promising Considering Their Incredible Capacity to Modify, Encapsulate and Co-Deliver Medicinal Compounds, Cells, Biomolecules, and Nanomaterials." https://doi.org/10.31219/osf.io/px3qy.

163. Moataz Dowaidar. 2021cc. "Immune Evasion Is Linked to Histone Variation Malfunction. Gene Therapy Could Provide Tools for Targeting Histone Variant Deposition as a Critical Part of Its Pharmacology." https://doi.org/10.31219/osf.io/kjm76.

164. Moataz Dowaidar. 2021cd. "Implementing the Human Artificial Chromosome Gene Therapy Platform Remains Challenging, but Continuous Animal Model Research Will Advance the Platform Closer to Clinical Trials." https://doi.org/10.31219/osf.io/a53f7. 
165. Moataz Dowaidar. 2021ce. "Inflammatory Breast Cancer Remains the Most Aggressive Form of Breast Cancer. A Multimodality Therapeutic Plan Has Shown Improved Survival Results.” https://doi.org/10.31219/osf.io/cr935.

166. Moataz Dowaidar. 2021cf. "Inherited Immunohematological and Metabolic Diseases Have the Potential to Improve Significantly, or Be Cured, Using Haematopoietic Stem Cell Transplantation Gene Therapy." https://doi.org/10.31219/osf.io/ukbnm.

167. Moataz Dowaidar. 2021cg. "Insulin and IGF-1 Receptors Mutations Can Lead to Targets for Gene Therapy in Diabetes, Obesity, and Metabolic Syndrome." https://doi.org/10.31219/osf.io/s86x5.

168. Moataz Dowaidar. 2021ch. "Integrating High-Throughput Genetics and Neuroimaging Technologies Promises Greater Information on Neurobiological Anomalies in Neurodegenerative Diseases." https://doi.org/10.31219/osf.io/hpgyz.

169. Moataz Dowaidar. 2021ci. "Intravitreal and Subretinal Injections Currently Deliver Most Gene Therapy, Including siRNA for Eye Illnesses. Non-Viral Vectors May Provide Targeting." https://doi.org/10.31219/osf.io/rjkhy.

170. Moataz Dowaidar. 2021cj. "LncRNA Regulating Reprogramming Glucose Metabolism Has Become One of the Most Tempting Antineoplastic Targets for Gene Therapy." https://doi.org/10.31219/osf.io/hqma5.

171. Moataz Dowaidar. 2021ck. "IncRNAs Are Upregulated and Downregulated in OS Cells. Angiogenesis, Metastasis, Cell Signaling, Autophagy, and Death Are among Biological Processes That RNAs Play a Role in." https://doi.org/10.31219/osf.io/48n7q.

172. Moataz Dowaidar. 2021cl. "Magnetic Nanoparticles Are Widely Used in Drug Delivery, Imaging, Diagnosis, and Targeting. It Has Promises for the Treatment of Inflammatory Disorders such as Rheumatoid Arthritis." https://doi.org/10.31219/osf.io/p2gme.

173. Moataz Dowaidar. 2021cm. "Many miRNAs Participate in Inflammatory Regulation and Bone Metabolism. Overexpression of miR21 and miR155 Releases Proinflammatory Cytokines." https://doi.org/10.31219/osf.io/2wuvp.

174. Moataz Dowaidar. 2021cn. "MiR490's Diagnostic Capacity Was Demonstrated in Various Cancer Kinds and Diseases, Adding to Its Clinical Value." https://doi.org/10.31219/osf.io/wysre.

175. Moataz Dowaidar. 2021co. "miRNAs Have an Impact on Xeno-Infectious Diseases by Influencing Host And/or Infection Factors." https://doi.org/10.31219/osf.io/7qewx.

176. Moataz Dowaidar. 2021cp. "Mutations in MED12 Lead to Mental Retardation, Including Opitz-Kaveggia Syndrome, Ohdo Syndrome, Lujan-Fryns Syndrome, and Psychosis. It's a Target for Gene Therapy." https://doi.org/10.31219/osf.io/cyns8.

177. Moataz Dowaidar. 2021cq. "Nanocarriers Can Be Used to Control the Activity of Genome Editing in a Spatiotemporal Way by Using Stimulusresponsive Nanocarriers." https://doi.org/10.31219/osf.io/nua89.

178. Moataz Dowaidar. 2021cr. "Nanomaterials Were Formed into Various Shapes, with Functionalization Aimed at Various Internalization Processes. Their Nanoscale Size Allows Drugs to Reach Cells or Extracellular Environments." https://doi.org/10.31219/osf.io/p2ajv. 
179. Moataz Dowaidar. 2021cs. "Nanomedicine Is Offering Promising Strategies for Tumor Blockade Treatment." https://doi.org/10.31219/osf.io/yzxuq.

180. Moataz Dowaidar. 2021ct. "Network Medicine Might Lead to New Treatments for Dyslipidemia. It Will Be a Challenging Method to Implement in a Clinical Context." https://doi.org/10.31219/osf.io/nksbw.

181. Moataz Dowaidar. 2021cu. "Neuroinflammation Caused by Activated Microglia and Astrocytes Can Contribute to the Progression of Pathogenic Damage to Substantia Nigra Neurons, Playing a Role in Parkinson's Disease Progression." https://doi.org/10.31219/osf.io/ac896.

182. Moataz Dowaidar. 2021cv. "Neurologists Rarely Perform Genetic Testing for Parkinson's Disease. Evidence Suggests That Many Patients with Major Genetic Variants Go Undiagnosed.” https://doi.org/10.31219/osf.io/ykpb2.

183. Moataz Dowaidar. 2021cw. "Neuronal Intranuclear Hyaline Inclusion Disease Is a Neurodegenerative Condition Which Can Be a Target for Gene Therapy." https://doi.org/10.31219/osf.io/upgqd.

184. Moataz Dowaidar. 2021cx. "New Therapies Aim at Restoring the Molecular, Morphological, and Functional Integrity of Parkinson's Specific Brain Circuits." https://doi.org/10.31219/osf.io/dvyxc.

185. Moataz Dowaidar. 2021cy. 'Not All lncMIRHGs Are 'Junk Transcripts,'. LncM IRHG Loci May Make Both Functional miRNAs and lncRNAs, Which Can Work Together or Separately." https://doi.org/10.31219/osf.io/a567w.

186. Moataz Dowaidar. 2021cz. "Nrf2 Signaling Pathways Are Part of a Wider Network of Signaling Pathways Regulating Thymoquinone Therapeutic Actions Which Need Innovative Formulations and Delivery Methods.” https://doi.org/10.31219/osf.io/u2fa7.

187. Moataz Dowaidar. 2021da. "Omics Should Be Integrated with Genomics to Uncover Molecular Networks and Tissue and Single-Cell Epigenetic Changes. With These Findings, Targeted Pseudoexfoliation Syndrome and Glaucoma Gene Therapy Procedures May Be Viable.” https://doi.org/10.31219/osf.io/48fj5.

188. Moataz Dowaidar. 2021db. "Ophthalmic Gene and Cell Therapies." https://doi.org/10.31219/osf.io/n84m9.

189. Moataz Dowaidar. 2021dc. "P21 Is a Flexible, Multi-Functional Protein. It Governs Various Tumor Cell Activities, Including Autophagy. p21 Is a Possible Radiotherapy Target." https://doi.org/10.31219/osf.io/ydkca.

190. Moataz Dowaidar. 2021dd. "Parkinson's Disease Simulating Complexity via Improving the Identification of Significant Genetic Alterations and Environmental Contaminants Should Be a Priority." https://doi.org/10.31219/osf.io/pmcu9.

191. Moataz Dowaidar. 2021de. "Patient-Specific Microphysiology Systems Are Likely to Become a Crucial Aspect of Translational Research and Precision Medicine." https://doi.org/10.31219/osf.io/bc8fr.

192. Moataz Dowaidar. 2021df. "Patients with PMD Who Are Thoroughly Screened by Genomic Medicine Have a Considerable Chance of Benefiting Greatly from Whole-Genome Sequencing.” https://doi.org/10.31219/osf.io/dajft. 
193. Moataz Dowaidar. 2021dg. "Polydopamine Nanoparticles' Activity and Long-Term Stability Should Be Fully Studied for Gene Therapy Applications." https://doi.org/10.31219/osf.io/x4nej.

194. Moataz Dowaidar. 2021dh. "Potential Therapeutics for Primary Mitochondrial Disorders." https://doi.org/10.31219/osf.io/6pz5k.

195. Moataz Dowaidar. 2021di. "Potentials of Medicinal Nanostructured Diamond Particles and Coatings." https://doi.org/10.31219/osf.io/h68xz.

196. Moataz Dowaidar. 2021dj. "Preclinical Investigations Revealed Possibilities for Salmonella Tumor Treatment. Bacteria Can Also Be Coupled to Nanomaterials Enabling Drug-Loading, Photocatalytic And/or Magnetic Properties, Using the Bacteria's Net Negative Charge." https://doi.org/10.31219/osf.io/embqk.

197. Moataz Dowaidar. 2021dk. "Research into P2X Purinergic Receptor Function in Tumor Growth Has Made Substantial Progress with Potential Gene Therapy Targeting." https://doi.org/10.31219/osf.io/r34fs.

198. Moataz Dowaidar. 2021dl. "RNA Therapies Hold Great Promise for Treating Cancer. High-Throughput Screening Techniques Have Facilitated the Development of RNA Treatments." https://doi.org/10.31219/osf.io/9vxrb.

199. Moataz Dowaidar. 2021dm. "RNAi Treatment Has Been Shown to Successfully Modify Human-Related Target Gene Expression, Including Cancer. It Has the Capacity to Control Non-Standard Oncogenes, such as Oncogenic lncRNAs." https://doi.org/10.31219/osf.io/bwqep.

200. Moataz Dowaidar. 2021dn. "RNAs Hold a Lot of Potential When It Comes to Druggable Molecular Targets." https://doi.org/10.31219/osf.io/2dtxg.

201. Moataz Dowaidar. 2021do. "Shadow Enhancers' Objective Seems to Be to Establish Robust Growth Patterns Independent of Genetic or Environmental Stress." https://doi.org/10.31219/osf.io/qfnkp.

202. Moataz Dowaidar. 2021dp. "Sickle Cell Disease Hematopoietic Stem Cell Gene Therapy with Globin Gene Addition Is Promising." https://doi.org/10.31219/osf.io/j5fkb.

203. Moataz Dowaidar. 2021dq. "Single-Gene Mutations in mtDNA-Associated Proteins Are Unlikely to Be the Main Cause of Sporadic Parkinson's Disease. Cumulative Genetic Variation in Numerous Genes May Be Important in Neurodegeneration and PD Risk." https://doi.org/10.31219/osf.io/89qte.

204. Moataz Dowaidar. 2021dr. "Small Nuclear Ribonucleoproteins (snRNPs) Based Gene Therapy." https://doi.org/10.31219/osf.io/c43r9.

205. Moataz Dowaidar. 2021ds. "Studying the Pathologic Mechanisms of Osteoporosis and the Bone Microenvironment May Help Researchers Better Know the Etiology of Rheumatoid Arthritis, Periodontitis, and Multiple Myeloma, as Well as Other Inflammatory and Autoimmune Disorders." https://doi.org/10.31219/osf.io/t3z6y.

206. Moataz Dowaidar. 2021dt. "Suicide Gene Therapy May Be Effective in the Treatment of Malignant Glioma.” https://doi.org/10.31219/osf.io/vdkst. 
207. Moataz Dowaidar. 2021du. "Synuclein Is a Protein That Is Expressed in Brain Tissue. The Specific Missense Mutation (SNCA) Found in a Family with Parkinson's Disease Is the Cause. Other Diseases Include Alzheimer's Disease and REM Sleep Behavior Disorder." https://doi.org/10.31219/osf.io/bs8rc.

208. Moataz Dowaidar. 2021dv. "Systems Biology Is a Method for Analyzing Massive Amounts of Multidimensional Data Generated by Omics Technologies. Cross-Validation of the Various Technological Platforms Is Critical." https://doi.org/10.31219/osf.io/p8vkd.

209. Moataz Dowaidar. 2021dw. "Targeting Mitochondria and Especially Taz Gene Mutation Induces CL May Give Novel Therapeutic Alternatives for Treating Barth Syndrome.” https://doi.org/10.31219/osf.io/unfpy.

210. Moataz Dowaidar. 2021dx. "The Ability to Combine Multiple mRNA Antigens Targeting Multiple Pathogens Simultaneously, and the Robust Immune Responses Are Confirmed in Several Clinical Studies." https://doi.org/10.31219/osf.io/6qksx.

211. Moataz Dowaidar. 2021dy. "The Cubic Polyhedral Oligomeric Silsesquioxanes Based Hybrid Materials Have a Wide Variety of Applications, Including Drug Administration, Gene Therapy, Biological Imaging, and Bone Regeneration." https://doi.org/10.31219/osf.io/9peq8.

212. Moataz Dowaidar. 2021dz. "The Development of Tissue Replacement Therapies and Drug Discovery Was a Critical Milestone in Advancing Regenerative Medicine." https://doi.org/10.31219/osf.io/w9bsm.

213. Moataz Dowaidar. 2021ea. "The Epidemic of COVID-19 Prompted Widespread Use of mRNA Vaccinations." https://doi.org/10.31219/osf.io/jqws5.

214. Moataz Dowaidar. 2021eb. "The Most Useful and Commonly Available Acute Rejection Surveillance Strategies Are Routine Monitoring of Myocardial Function and Donor-Specific Anti-HLA Abs Monitoring." https://doi.org/10.31219/osf.io/ebw68.

215. Moataz Dowaidar. 2021ec. "The Protease MBTPS2 Is an Important Regulator of Several Cellular Processes, Especially in Health and Sickness." https://doi.org/10.31219/osf.io/qyn6h.

216. Moataz Dowaidar. 2021ed. "The Sigma 1 Receptor (S1R) Is a Potential Therapeutic Target for the Treatment of Huntington's Disease." https://doi.org/10.31219/osf.io/mcefx.

217. Moataz Dowaidar. 2021ee. "The Use of a Network Medicine Approach Might Result in Innovative Strategies for Lowering Coronary Heart Disease and CV Risks." https://doi.org/10.31219/osf.io/eakg8.

218. Moataz Dowaidar. 2021ef. "The Vasoconstrictor Endothelin System Involvement in Chronic Kidney Diseases Pathogenesis Is Now the Most Often Employed Treatment Method." https://doi.org/10.31219/osf.io/cnkqy.

219. Moataz Dowaidar. 2021eg. "The VPS35-D620N Mutation Is Associated with Parkinson's Disease and Can Be a Target for Gene Therapy." https://doi.org/10.31219/osf.io/83sxr.

220. Moataz Dowaidar. 2021eh. "Therapeutics Including Gene Therapy for Osteoarthritis as a Concept." https://doi.org/10.31219/osf.io/7zsqy. 
221. Moataz Dowaidar. 2021ei. "Tissue Hypoxia Has Been Established as a Master Regulator for Alternative Splicing, with Substantial Clinical Consequences and Possibilities for Gene Therapy Targeting." https://doi.org/10.31219/osf.io/5pbw4.

222. Moataz Dowaidar. 2021ej. "To Rectify Alzheimer's Disease Etiology, Excessive Mitochondrial Division Might Be Stopped or Mitophagy Might Be Promoted." https://doi.org/10.31219/osf.io/6kdxw.

223. Moataz Dowaidar. 2021ek. "Transcriptomics Is a Rapidly Growing Field That Generates New Data That May Be Used on Its Own or in Combination with Existing Clinical Data for Development of New Therapeutics, Including Gene Therapy." https://doi.org/10.31219/osf.io/kfr6a.

224. Moataz Dowaidar. 2021el. "Tumor Microenvironment Has Clinical Significance in Terms of Prognosis and Therapy Prediction.” https://oi.org/10.31219/osf.io/4dz8q.

225. Moataz Dowaidar. 2021em. "Using AAV as a Gene Delivery Vector in the Neural System Is Effective in Several Animals, such as Nonhuman Primates." https://doi.org/10.31219/osf.io/ut4fa.

226. Moataz Dowaidar. 2021en. "Using Pre-Existing Datasets to Combine Published Information with New Metrics Would Help Researchers Construct a Broader Picture of Chromatin in Disease." https://doi.org/10.31219/osf.io/gsqv5.

227. Moataz Dowaidar. 2021eo. "Virus-like Particles Are Good Nanocarriers for Liquid Biopsy Probes, Imaging Contrast Agents, and Anticancer Medications." https://doi.org/10.31219/osf.io/xbtka.

228. Moataz Dowaidar. 2021ep. "ZEB1 Controls the Expression of ICAM1, Promoting Monocyte-Macrophage Adhesion and Hence the Formation of Atherosclerotic Lesions." https://doi.org/10.31219/osf.io/kzjqg.

229. Moataz Dowaidar. 2021eq. "Gene Therapy Development and Legislation." https://doi.org/10.31219/osf.io/mwb2n.

230. Moataz Dowaidar. 2021er. "Next-Generation Sequencing Is Now Utilized to Identify Genetic Abnormalities and Develop Gene Therapy." https://doi.org/10.31219/osf.io/em7xp.

231. Moataz Dowaidar. 2021es. "Nucleic Acid Designs, Artificial Intelligence for Screening Nanomaterials, and Enhanced Characterization Methods Are Needed to Make Nanomedicine More Successful." https://doi.org/10.31219/osf.io/2w5aq.

232. Moataz Dowaidar. 2021et. "Potential Strategies for Cancer Gene Therapy." https://doi.org/10.31219/osf.io/atcqz.

233. Moataz Dowaidar. 2021eu. "Quantitative Groups Will Be Critical to the Success of Future Gene Therapy Programs.” https://doi.org/10.31219/osf.io/v97ht.

234. Moataz Dowaidar. 2021ev. "The Treatment of Major Human Illnesses with Recombinant Adeno-Associated Virus (rAAV) Has Shown Tremendous Promises." https://doi.org/10.31219/osf.io/uwa4e.

235. Moataz Dowaidar. 2021ew. "Carbon Nanotubes Have Enormous Potential in Gene Therapy.” https://doi.org/10.31219/osf.io/9bcxk.

236. Moataz Dowaidar. 2021ex. "Charge-Alteration-Based Approaches Can Address the Evolving Needs of Nucleic Acid-Based Gene Therapy, Charge Reversal Techniques Are Also Promising." https://doi.org/10.31219/osf.io/zwq5h. 
237. Moataz Dowaidar. 2021ey. "Chromosome X, the Most Explored Genome-Editing Chromosome, Presents Possibilities for Hemophilia A Treatments." https://doi.org/10.31219/osf.io/6vsdz.

238. Moataz Dowaidar. 2021ez. "Clinical Investigations Show That siRNA May Be Used to Treat a Variety of Disorders, Including Cancer." https://doi.org/10.31219/osf.io/fcsgq.

239. Moataz Dowaidar. 2021fa. "Cyclodextrins as Potential Gene Therapy Vectors." https://doi.org/10.31219/osf.io/zhtsc.

240. Moataz Dowaidar. 2021fb. "Development of Specialized Carriers Capable of Delivering Effective RNAi and siRNA Gene Therapy." https://doi.org/10.31219/osf.io/3ykwm.

241. Moataz Dowaidar. 2021fc. "Gene Therapy Can Target Mutations such as BRAF, Which Have Been Shown to Make Tumors More Susceptible to Autophagy Suppression." https://doi.org/10.31219/osf.io/3gwra.

242. Moataz Dowaidar. 2021fd. "Gene Therapy Vectors Should Enable CRISPR Systems to Accumulate at Disease Sites and Successfully Penetrate Nuclei." https://doi.org/10.31219/osf.io/xzmnc.

243. Moataz Dowaidar. 2021fe. "Nanoformulations Can Be Utilized to Deliver Effective siRNA to Tumor Cells to Decrease Gene Expression." https://doi.org/10.31219/osf.io/zvukc.

244. Moataz Dowaidar. 2021ff. "Neuronal Ceroid Lipofuscinosis Therapeutics." https://doi.org/10.31219/osf.io/75vcp.

245. Moataz Dowaidar. 2021fg. "Nonviral Gene Delivery Vectors for Transfection of the CAR Gene for CAR-T Cell Therapy." https://doi.org/10.31219/osf.io/ckxh5.

246. Moataz Dowaidar. 2021fh. "Potential HIV Gene Therapy Strategies." https://doi.org/10.31219/osf.io/e5hm2.

247. Moataz Dowaidar. 2021fi. "Research on Cell Sources for Brain Cell Replacement Methods Has Gained Major Importance. Cell and Gene Therapy Are Potentially Intriguing New Domains of Regenerative Medicine.” https://doi.org/10.31219/osf.io/g835b.

248. Moataz Dowaidar. 2021fj. "RNAi-Based Gene Therapy Provides a Wide Variety of Applications. Safe, Biodegradable Nano Delivery Vectors Are Still Needed." https://doi.org/10.31219/osf.io/s2zhn.

249. Moataz Dowaidar. 2021fk. "Strategies for Treating Multiple Sclerosis with Gene Therapy." https://doi.org/10.31219/osf.io/sycn6.

250. Moataz Dowaidar. 2021fl. "The Combination of Unique Biomolecules and Nanoparticles Has Shown Successful Gene Therapy Treatment Approaches for Non-Small Cell Lung Cancer Treatment." https://doi.org/10.31219/osf.io/yeq5z.

251. Moataz Dowaidar. 2021fm. "Understanding Why the Same Gene Delivery Vector Behaves Differently in Different Cell Types Is Essential for Developing More Adaptable Transfection Systems." https://doi.org/10.31219/osf.io/6q8af. 
252. Moataz Dowaidar. 2021fn. "AAV9 Is Considered the Most Efficient AAV Serotype Targeting Blood-Brain Barriers. To Enhance Effective Gene Therapy for CNS Illnesses, Testing Novel Vectors with More Efficient Crossing Capabilities Is Vital." https://doi.org/10.31219/osf.io/7bf5s.

253. Moataz Dowaidar. 2021fo. "Artificial miRNAs Are Potential Gene Therapy Tools, Especially for Incurable Monogenic Disorders." https://doi.org/10.31219/osf.io/d5rnm.

254. Moataz Dowaidar. 2021fp. "Breakthroughs in mRNA Modification and Nanoparticle-Based Delivery Vehicles Facilitate Gene Therapy Strategies." https://doi.org/10.31219/osf.io/ky7dt.

255. Moataz Dowaidar. 2021fq. "CRISPR/Cas9-Mediated Genome Editing Has Demonstrated Significant Promise for Genetic Correction in Autologous Hematopoietic Stem/progenitor Cells (HSPCs) and Induced Pluripotent Stem Cells (iPSCs)." https://doi.org/10.31219/osf.io/xk54r.

256. Moataz Dowaidar. 2021fr. "Gene Therapy Vectors for Targeting the Heart." https://doi.org/10.31219/osf.io/gcbhf.

257. Moataz Dowaidar. 2021fs. "Liposomes Can Minimize Cardiotoxicity, Address Drug Resistance, and Improve Overall Drug Release Profiles in Breast Cancer." https://doi.org/10.31219/osf.io/tn56d.

258. Moataz Dowaidar. 2021ft. "Liposomes with Cerasome-Forming Lipids as Gene Therapy Vectors.” https://doi.org/10.31219/osf.io/zjn6v.

259. Moataz Dowaidar. 2021fu. "Nanomaterials Combine Multiple Therapeutic Approaches for Cancer Cell Multidrug Resistance, Ferroptotic Cell Death Is Promising in Various Cancers." https://doi.org/10.31219/osf.io/7bg9t.

260. Moataz Dowaidar. 2021fv. "Nanomedicines for Enhanced Permeability and Retention (EPR)-Stratified Patients Have the Potential to Improve Treatment Outcomes." https://doi.org/10.31219/osf.io/xrcb2.

261. Moataz Dowaidar. 2021fw. "RNA-Based Gene Therapy for Manipulating the Neuroinflammatory Cascade Closely Linked to Neurodegeneration Can Help Reduce Disease Development." https://doi.org/10.31219/osf.io/2hswv.

262. Moataz Dowaidar. 2021fx. "Targeted Chemical Nucleases Have a Wide Range of Untapped Applications in Biological Fields, Including Gene Therapy." https://doi.org/10.31219/osf.io/6bexs.

263. Moataz Dowaidar. 2021fy. "Bacterial Nanoparticles Can Deliver Proteins, Medications, Enzymes, and Genes to Diagnose and Cure Numerous Illnesses." https://doi.org/10.31219/osf.io/7gyna.

264. Moataz Dowaidar. 2021fz. "Exosomal miRNA Diagnostic and Gene Therapy Tools." https://doi.org/10.31219/osf.io/aknrc.

265. Moataz Dowaidar. 2021ga. "Gene Modification Research Has Potential, from Diagnostic to Therapeutic Levels. The Most Promising Metabolic Pathways Include the TGF-1 Signaling System, Inflammation and Protein Transport." https://doi.org/10.31219/osf.io/5ert4.

266. Moataz Dowaidar. 2021gb. "Gene Therapy Using MnO2 Nanoparticles." https://doi.org/10.31219/osf.io/xmwjs. 
267. Moataz Dowaidar. 2021gc. "Gene-Regulatory Elements May Change the Amount, Timing, or Location of Gene Expression, Cis-Regulation Therapy Platforms Might Become a Gene Therapy to Treat Many Genetic Diseases." https://doi.org/10.31219/osf.io/xc5a2.

268. Moataz Dowaidar. 2021gd. "Hemophilia Therapeutics." https://doi.org/10.31219/osf.io/gu74x.

269. Moataz Dowaidar. 2021ge. "Mesenchymal Stem Cells Strategies in Cancer Immunotherapy." https://doi.org/10.31219/osf.io/dkv6w.

270. Moataz Dowaidar. 2021gf. "Nanomaterials Can Inhibit Planktonic and Biofilm Bacteria and Can Be Used as Topical Therapy for Mouth and Wound-Related Infections." https://doi.org/10.31219/osf.io/aqd2e.

271. Moataz Dowaidar. 2021gg. "New Technologies to Improve CAR T Cell Generation and Biomanufacturing Will Lead to Safer, More Therapeutically Effective Cells." https://doi.org/10.31219/osf.io/un8gp.

272. Moataz Dowaidar. 2021gh. "Ocular Gene Therapy Strategies." https://doi.org/10.31219/osf.io/7en3k.

273. Moataz Dowaidar. 2021gi. "Peripheral Nerve Injury Therapeutics, Including Electrical Stimulation, Stem Cell Treatments, and Synthetic Neural Scaffolds, Have Shown Promising Preclinical and Even Clinical Results with Potential Regenerative Treatment." https://doi.org/10.31219/osf.io/m8cs9.

274. Moataz Dowaidar. 2021gj. "Photothermal and Photodynamic Photoactivation of Nanomaterials-Based Prodrugs Are Two Key Methods for NIR Light-Mediated Photoactivation." https://doi.org/10.31219/osf.io/2bh3r.

275. Moataz Dowaidar. 2021gk. "Quantum Dots Have the Potential to Be Used in Gene Therapy." https://doi.org/10.31219/osf.io/bdeg6.

276. Moataz Dowaidar. 2021gl. "Sickle Cell Disease Has Emerged as a Public Health Concern. Some Drugs May Conflict with Curative Therapies, yet They May Be Useful as a Bridge to HSCT and Gene Therapy." https://doi.org/10.31219/osf.io/6kufh.

277. Moataz Dowaidar. 2021gm. "Stimulator of Interferon Genes (STING)-Activating Nanoparticles Can Be Employed as a Tool for Controlled Immune Activation." https://doi.org/10.31219/osf.io/2ez7a.

278. Moataz Dowaidar. 2021gn. "CRISPR/Cas9 Has Introduced New Gene Therapy Possibilities for Muscular Dystrophies.” https://doi.org/10.31219/osf.io/ug8v4.

279. Moataz Dowaidar. 2021go. "Degradable Branched Polycationic Systems Are Promising Gene Therapy Vectors.” https://doi.org/10.31219/osf.io/utypf.

280. Moataz Dowaidar. 2021gp. "Developing Nanotechnology Platforms for Peptide-Based Combinatory Cancer Gene Therapy Will Likely Have a Significant Influence on the Development of Personalized Cancer Medicines." https://doi.org/10.31219/osf.io/zbrkj.

281. Moataz Dowaidar. 2021gq. "Exosomes May Prevent Cardiac Attacks, Heart Failure, and Cardiomyopathy." https://doi.org/10.31219/osf.io/agm3k.

282. Moataz Dowaidar. 2021gr. "Exosomes Potential Therapeutics." https://doi.org/10.31219/osf.io/mhwt3. 
283. Moataz Dowaidar. 2021gs. "Gene Therapy Using miRNA Treatment Suppresses the Expression of Bone-Forming Defective Genes and Raises the Expression of Genes That Become Dormant during Bone Building." https://doi.org/10.31219/osf.io/tcka3.

284. Moataz Dowaidar. 2021gt. "Genome-Editing Is Promising for Producing Therapeutically Relevant Animal Models for Possible Therapies for Rare Human Diseases." https://doi.org/10.31219/osf.io/dehr9.

285. Moataz Dowaidar. 2021gu. "Human Corneal Endothelial Cells Grafts to Replace Cadaveric Donor Corneas." https://doi.org/10.31219/osf.io/p9x7e.

286. Moataz Dowaidar. 2021gv. "Hybrid Nanotechnology and Peptide Nucleic Acid Could Improve the Effectiveness of Gene Therapy by Increasing Its Cell Permeability." https://doi.org/10.31219/osf.io/d8wzt.

287. Moataz Dowaidar. 2021gw. "In Prenatal Stem Cell Transplantation and in Utero Gene Therapy, a Wide Spectrum of Genetic Diseases Can Be Diagnosed and Treated before Birth.” https://doi.org/10.31219/osf.io/sa3vz.

288. Moataz Dowaidar. 2021gx. "Magnetic Iron Oxide Nanoparticles Have Potential on Gene Therapy Effectiveness and Biocompatibility." https://doi.org/10.31219/osf.io/f3hm4.

289. Moataz Dowaidar. 2021gy. "Neurotrophin Gene Therapy May Be Able to Treat Individuals with Noise-Induced Hearing Loss or Neural Presbyacusis." https://doi.org/10.31219/osf.io/spkxh.

290. Moataz Dowaidar. 2021gz. "Plant Viral Nanoparticles Can Be Used in Biological Systems for Loading and Transporting Cargo." https://doi.org/10.31219/osf.io/txdka.

291. Moataz Dowaidar. 2021ha. "Polydopamine May Be Easily Functionalized with a Range of Nanomaterials for Synergistic Cancer Therapy, in Addition to Its Exceptional Photothermal Effects.” https://doi.org/10.31219/osf.io/cq942.

292. Moataz Dowaidar. 2021hb. "Tumor-Targeted Drug Delivery Systems for Anticancer Therapies Can Selectively Provide an Appropriate Cytotoxic Payload to Cancer Cells, Reducing the Side Effects of Chemo." https://doi.org/10.31219/osf.io/683nj.

293. Moataz Dowaidar. n.d. "Guidelines for the Use and Interpretation of Assays for Monitoring Autophagy (4th Edition)."

294. Dowaidar, Moataz, Hani Nasser Abdelhamid, Mattias Hällbrink, Krista Freimann, Kaido Kurrikoff, Xiaodong Zou, and Ülo Langel. 2017. "Magnetic Nanoparticle Assisted Self-Assembly of Cell Penetrating Peptides-Oligonucleotides Complexes for Gene Delivery.” $\quad$ Scientific $\quad$ Reports $7 \quad$ (1): 9159. https://doi.org/10.1038/s41598-017-09803-z.

295. Dowaidar, Moataz, Hani Nasser Abdelhamid, Mattias Hällbrink, Ülo Langel, and Xiaodong Zou. 2018. "Supplemental Material for Chitosan Enhances Gene Delivery of Oligonucleotide Complexes with Magnetic Nanoparticles-cell-Penetrating Peptide." SAGE Journals. https://doi.org/10.25384/SAGE.7105436.V1.

296. Dowaidar, Moataz, Hani Nasser Abdelhamid, Mattias Hällbrink, Xiaodong Zou, and Ülo Langel. 2017a. "Graphene Oxide Nanosheets in Complex with Cell Penetrating Peptides for Oligonucleotides Delivery General Subjects." Biochimica et Biophysica Acta, General Subjects. https://pubag.nal.usda.gov/catalog/5734174. 
297. Moataz Dowaidar. 2017b. "Graphene Oxide Nanosheets in Complex with Cell Penetrating Peptides for Oligonucleotides Delivery." Biochimica et Biophysica Acta, General Subjects 1861 (9): 2334-41. https://doi.org/10.1016/j.bbagen.2017.07.002.

298. Dowaidar, Moataz, and Moataz Dowaidar. 2018. "Chimeric Gene Delivery Vectors : Design, Synthesis, and Mechanisms from Transcriptomics Analysis."

299. Moataz Dowaidar. 2021a. "Addiction Biology Research on miRNAs, and Their Role in the Pathophysiology of Addiction Is Enabling Gene Therapy Opportunities." https://doi.org/10.31219/osf.io/z5wyt.

300. Moataz Dowaidar. 2021b. "Aptamers Targeting Vascular Endothelial Growth Factor Molecular Regulation as Potential Therapists." https://doi.org/10.31219/osf.io/a8qpr.

301. Moataz Dowaidar. 2021c. "Arrhythmogenic Cardiomyopathy Is a Set of Hereditary Cardiac Muscle Disorders Where Various Etiologies Converge. Most ACM Patients Do Not Have a Genetic Diagnosis.” https://doi.org/10.31219/osf.io/pztv3.

302. Moataz Dowaidar. 2021d. "Autophagy, Immunological Response, and Inflammation All Rely on the TRIM Family Proteins. TRIM-Based Therapeutics for Inflammatory Illnesses Including Diabetes and Diabetic Comorbidities Are Promising." https://doi.org/10.31219/osf.io/y4g6e.

303. Moataz Dowaidar. 2021e. "Biogenic Particles Can Be Multiantigenic, Immunostimulative and Activate Innate Immunity While Suppressing Tumor Development." https://doi.org/10.31219/osf.io/q2kby.

304. Moataz Dowaidar. 2021f. "Biological Medications for Interventional Pain Have a Lot of Clinical Data behind Them. It Is Fair to Assume They Will Replace Steroid-Based Interventional Techniques, Providing Patients with Longer Relief." https://doi.org/10.31219/osf.io/4y5fm.

305. Moataz Dowaidar. 2021g. "Carbon Nanofibers Assist in the Manufacture of Prosthetic Joints, Promote Tissue, Organ, Nerve Regeneration and Development, and Improve Anticancer Therapy Impact and Chemosensitization for a Range of Tumor Types." https://doi.org/10.31219/osf.io/z3ucn.

306. Moataz Dowaidar. 2021h. "Emerging Therapy Options May Help Patients with RAG Deficiency, Especially Those with Severe Immune Dysregulation." https://doi.org/10.31219/osf.io/v5tjg.

307. Moataz Dowaidar. 2021i. "Exosomes as Promising Gene Therapy Tools Still Need to Be Researched and Manufactured More Efficiently." https://doi.org/10.31219/osf.io/nw4z7.

308. Moataz Dowaidar. 2021j. "Focus on Exosomes Could Help Make the Use of Circulating miRNA as Biomarkers More Practical. A Detailed Understanding of miRNA Behavior Should Be a Subject of Gene Therapy." https://doi.org/10.31219/osf.io/uan6x.

309. Moataz Dowaidar. 2021k. "Gene-Free Viral-like Particles (VLPs) Offer a Safer Alternative to Inactivating or Weakening Viral Strains for Traditional Vaccines. VLP-Based Vaccinations without Adjuvants Have Been Found to Promote Humoral and Cellular Immunity.” https://doi.org/10.31219/osf.io/9dvut. 
310. Moataz Dowaidar. 20211. "Given the Importance of mTOR Signaling in a Number of Illnesses, It Looks Suitable to Use miR 99 Family Members as a Therapeutic Intervention to Deal with These Illnesses by Using Gene Therapy Tools." https://doi.org/10.31219/osf.io/8cwgh.

311. Moataz Dowaidar. 2021m. "HMGB1 Has Sparked a Lot of Attention as a Model DAMP Molecule Involved in Inflammation, Inflammatory Diseases, and Cancer." https://doi.org/10.31219/osf.io/5qx36.

312. Moataz Dowaidar. 2021n. "Nucleic Acid Nanocarriers Can Be Programmable, Spatially Adjustable and Biocompatible, Minimizing Systemic Toxicity and Improving Pharmacodynamics." https://doi.org/10.31219/osf.io/wr237.

313. Moataz Dowaidar. 2021o. "Osteoporosis Is a Prominent Source of Morbidity and Mortality in the Elderly, Particularly in Postmenopausal Women. Long Noncoding RNAs (lncRNAs) Have Been Found to Be Important Regulators and Possible Gene Therapy Targets." https://doi.org/10.31219/osf.io/ghfpt.

314. Moataz Dowaidar. 2021p. "Polycomb Genes Role in Cancer Pathophysiology Is Offering Targets for Therapeutics Including Gene Therapy." https://doi.org/10.31219/osf.io/sfvej.

315. Moataz Dowaidar. 2021q. "RNA Sequencing and Microarray Analysis Are Helpful Techniques to Detect Obesity-Related lncRNAs. LncRNA Can Alter Cholesterol Metabolism and Can Be a Target for Gene Therapy." https://doi.org/10.31219/osf.io/3fb6w.

316. Moataz Dowaidar. 2021r. "Sepsis-Associated Acute Kidney Damage Is a Disease That Affects the Patient's Quality of Life. It Should Be a Target for Gene Therapy." https://doi.org/10.31219/osf.io/49k7q.

317. Moataz Dowaidar. 2021s. "The Gene Expression Profiling Gives an in-Depth Insight of Breast Cancer Heterogeneity, Better than a Single Protein or Gene Expression. It Is Time to Include It in the Daily Routine." https://doi.org/10.31219/osf.io/xhyd7.

318. Moataz Dowaidar. 2021t. "The Nanomedicine System Has Successfully Inhibited Tumor Neovascularization Using Gene Silencing, Chemotherapy, Photothermal Therapy, and Other Therapies." https://doi.org/10.31219/osf.io/rk2bf.

319. Moataz Dowaidar. 2021u. "The Therapeutic Application of a Nucleic Acid Sequence to Patients' Diseased Organs Is Currently Available." https://doi.org/10.31219/osf.io/pqsbf.

320. Moataz Dowaidar. 2021v. "Triple-Negative Breast Cancer, Which Lacks the Expression of Hormone Receptors and HER2, Has a Worse Prognosis. Massive Parallel Sequencing Is Capable of Reliably Breaking down the Intra-Tumor and Inter-Tumor Heterogeneity." https://doi.org/10.31219/osf.io/pvk7u.

321. Dowaidar, Moataz, Maxime Gestin, Carmine Pasquale Cerrato, Mohammed Hakim Jafferali, Helerin Margus, Paula Ann Kivistik, Kariem Ezzat, et al. 2017. "Role of Autophagy in Cell-Penetrating Peptide Transfection Model." Scientific Reports 7 (1): 12635. https://doi.org/10.1038/s41598-017-12747-z. 
322. Dowaidar, Moataz, H. A. Ismail, A. A. Alghasham, M. M. Dowaidar, and A. A. Settin. 2011. "Polymorophisms in MTHF and Ace Genes and the Association with Hypertension among Saudi Population from Qassim Region.” Egyptian Journal of $\begin{array}{llll}\text { Biochemistry and } \quad \text { Molecular } & \text { Biology }\end{array}$ https://doi.org/10.4314/ejbmb.v29i1.67382.

323. Dowaidar, Moataz, Hani Nasser Abdelhamid, Mattias Hällbrink, Ülo Langel, and Xiaodong Zou. 2018. "Chitosan Enhances Gene Delivery of Oligonucleotide Complexes with Magnetic Nanoparticles-Cell-Penetrating Peptide." Journal of $\begin{array}{llll}\text { Biomaterials } \quad \text { Applications } & 33 & \text { (3): }\end{array}$ https://doi.org/10.1177/0885328218796623.

324. Dowaidar, Moataz, and Ahmad Settin. 2010. "Risk of Myocardial Infarction Related to Factor V Leiden Mutation: A Meta-Analysis." Genetic Testing and Molecular Biomarkers 14 (4): 493-98. https://doi.org/10.1089/gtmb.2010.0017.

325. Gestin, Maxime, Moataz Dowaidar, and Ülo Langel. 2017. "Uptake Mechanism of Cell-Penetrating Peptides." Advances in Experimental Medicine and Biology 1030: 255-64. https://doi.org/10.1007/978-3-319-66095-0_11.

326. Ismail, H. A., A. A. Alghasham, M. M. Dowaidar, and A. A. Settin. 2011. "Polymorophisms in MTHF and Ace Genes and the Association with Hypertension among Saudi Population from Qassim Region.” Egyptian Journal of Biochemistry and Molecular Biology 29 (1). https://doi.org/10.4314/ejbmb.v29i1.67382.

327. Settin, Ahmad A., Abdullah Algasham, Moataz Dowaidar, and Hisham Ismail. 2009. "Methylene Tetrahydrofolate Reductase and Angiotensin Converting Enzyme Gene Polymorphisms Related to Overweight/obesity among Saudi Subjects from Qassim Region.” Disease Markers 27 (2): 97-102. https://doi.org/10.3233/DMA-2009-0660.

328. Settin, Ahmad A., Abdullah Alghasham, Ahmad Ali, Moataz Dowaidar, and Hisham Ismail. 2012. "Frequency of Thrombophilic Genetic Polymorphisms among Saudi Subjects Compared with Other Populations." Hematology 17 (3): 176-82. https://doi.org/10.1179/102453312X13376952196575.

329. Settin, Ahmad, Ibrahem S. Abu-Saif, Rizk El-Baz, Moataz Dowaidar, Rabab Abu-Al Kasim, and Shaimaa Shabana. 2007a. "Diagnosis of Sex Chromosome Disorders and Prenatal Diagnosis of Down Syndrome Using Interphase Fluorescent In-Situ Hyperidization Technique." International Journal of Health Sciences 1 (2): 203-9. https://www.ncbi.nlm.nih.gov/pubmed/21475429.

330. Settin, Ahmad, Abdullah Algasham, Moataz Dowaidar, and Hisham Ismail. 2011. "Methylene Tetrahydrofolate Reductase (MTHFR) and Angiotensinogen Converting Enzyme (ACE) Gene Polymorphisms Related to Overweight and Obesity among Saudi Patients in Al Qassim.” International Journal of Health Sciences 5 (2 Suppl 1): 24-25. https://www.ncbi.nlm.nih.gov/pubmed/23284565.

331. Settin, Ahmad, Hala Almarsafawy, Ahmad Alhussieny, and Moataz Dowaidar. 2008a. "Dysmorphic Features, Consanguinity and Cytogenetic Pattern of Congenital Heart Diseases: A Pilot Study from Mansoura Locality, Egypt." International Journal of Health Sciences 2 (2): 101-11. https://www.ncbi.nlm.nih.gov/pubmed/21475491. 
332. Settin, Ahmad, Moataz Dowaidar, Rizk El-Baz, Ayman Abd-Al-Samad, Ibrahim El-Sayed, and Mahmoud Nasr. 2008. "Frequency of Factor V Leiden Mutation in Egyptian Cases with Myocardial Infarction." Hematology 13 (3): 170-74. https://doi.org/10.1179/102453308X316158.

333. Venit, Tomas, Moataz Dowaidar, Maxime Gestin, Syed Raza Mahmood, Ülo Langel, and Piergiorgio Percipalle. 2020. "Transcriptional Profiling Reveals Ribosome Biogenesis, Microtubule Dynamics and Expression of Specific lncRNAs to Be Part of a Common Response to Cell-Penetrating Peptides." Biomolecules 10 (11): 1567. https://doi.org/10.3390/biom10111567. 